\title{
Flow decomposition for heat equations with memory
}

\author{
Gengsheng Wang* $\quad$ Yubiao Zhang ${ }^{\dagger} \quad$ Enrique Zuazua ${ }^{\ddagger}$
}

\begin{abstract}
We build up a decomposition for the flow generated by the heat equation with a real analytic memory kernel. It consists of three components: The first one is of parabolic nature; the second one gathers the hyperbolic component of the dynamics, with null velocity of propagation; the last one exhibits a finite smoothing effect. This decomposition reveals the hybrid parabolic-hyperbolic nature of the flow and clearly illustrates the significant impact of the memory term on the parabolic behavior of the system in the absence of memory terms.
\end{abstract}

Keywords. Heat equations with memory, decomposition of the flow, hybrid parabolichyperbolic behavior

\section{Introduction}

\subsection{Statement of the problem}

In this paper, we will study the following heat equation with memory:

$$
\left\{\begin{array}{lc}
\partial_{t} y(t, x)-\Delta y(t, x)+\int_{0}^{t} M(t-s) y(s, x) d s=0, & (t, x) \in \mathbb{R}^{+} \times \Omega, \\
y(t, x)=0, & (t, x) \in \mathbb{R}^{+} \times \partial \Omega \\
y(0, x)=y_{0}(x), & x \in \Omega .
\end{array}\right.
$$

Here, $\mathbb{R}^{+}:=(0,+\infty), \Omega \subset \mathbb{R}^{n}\left(n \in \mathbb{N}^{+}:=\{1,2,3, \cdots\}\right)$ is a bounded domain with a $C^{2}$ boundary $\partial \Omega, y_{0}$ is an initial datum and $M$ is a memory-kernel over $\overline{\mathbb{R}^{+}}:=[0,+\infty)$.

Although our analysis can be generalized to less regular memory kernels, for the sake of simplicity we assume that:

(C) the memory kernel $M$ is a real analytic and nonzero function over $\overline{\mathbb{R}^{+}}$.

\footnotetext{
*Center for Applied Mathematics, Tianjin University, Tianjin, 300072, China. e-mail: wanggs62@yeah.net

${ }^{\dagger}$ Center for Applied Mathematics, Tianjin University, Tianjin, 300072, China; e-mail: yubiao_zhang@yeah.net

¥[1] Chair for Dynamics, Control and Numerics - Alexander von Humboldt-Professorship, Department of Data Science, Friedrich-Alexander-Universität Erlangen-Nürnberg, 91058 Erlangen, Germany (e-mail: enrique.zuazua@fau.de),

[2] Chair of Computational Mathematics, Fundación Deusto, Av. de las Universidades, 24, 48007 Bilbao, Basque Country, Spain,

[3] Departamento de Matemáticas, Universidad Autónoma de Madrid, 28049 Madrid, Spain

Mathematics Subject Classification (2010): 45K05 35K05 93C05
} 
Equations with memory arise in the modeling of many physical phenomena such as viscoelasticity, heat conduction, etc. They can be traced back to the works of J. Maxwell [14], L. Boltzmann $[2,3]$ and V. Volterra [19, 20]. For instance, in the analysis of elastic materials, L. Boltzmann and V. Volterra represented the stress tensor in terms of the strain tensor as well as its history values. Equations involving memory terms have been widely studied: see for instance $[1,4,5,6,7,8,9,10,11,13,17]$ and the references therein. In particular, in [11] the general memory effect in heat conduction processes was analyzed showing that temperature waves travelling in the direction of the heat-flux propagate faster than wave travelling in the opposite direction, while in [8] the asymptotic behavior of the systems of linear viscoelasticity at large time was analyzed, introducing a new auxiliary variable to deal with the history of the states.

By standard methods (see, for instance, [18, Theorem 1.2 in Section 6.1, p. 184]), it can be shown that the equation $(1.1)$, with $y_{0} \in L^{2}(\Omega)$, has a unique mild solution, denoted by $y\left(\cdot ; y_{0}\right)$, in the space $C\left(\overline{\mathbb{R}^{+}} ; L^{2}(\Omega)\right)$. For each $t \geq 0$, we let the evolution of the system be denoted by:

$$
\Phi(t) y_{0}:=y\left(t ; y_{0}\right), \quad y_{0} \in L^{2}(\Omega) .
$$

For each $t \geq 0$, the flow generated by the equation $(1.1), \Phi(t)$, belongs to $\mathcal{L}\left(L^{2}(\Omega)\right)$. Here and in what follows, we denote by $\mathcal{L}(E, F)$ (where $E$ and $F$ are two Banach spaces) the space of all linear and bounded operators from $E$ to $F$, and simply write $\mathcal{L}(E)$ for $\mathcal{L}(E, E)$.

We shall use the notation $\left\{e^{t A}\right\}_{t \geq 0}$ for the $C_{0}$ semigroup generated by the heat equation in the absence of memory term (i.e., when $M \equiv 0$ ), where

$$
A f:=\Delta f, \text { with its domain } D(A):=H^{2}(\Omega) \cap H_{0}^{1}(\Omega) .
$$

Then $z\left(t ; y_{0}\right):=e^{t A} y_{0}, t \geq 0$, solves (1.1) without memory, i.e., (1.1) when $M \equiv 0$.

This paper is devoted to analyzing the dynamics of the system with memory term and, in particular, to exhibiting the significant differences with the heat semigroup in the absence of memory.

\subsection{Main results}

The aim of this paper is to build up a decomposition of the flow $\Phi(t)$, revealing a hybrid parabolic-hyperbolic dynamics of the (1.1).

To state our main results, we first introduce several concepts, definitions and notations.

- Let $\eta_{j}>0$ be the $j^{\text {th }}$ eigenvalue of $-A$ and let $e_{j}$ be the corresponding normalized eigenfunction in $L^{2}(\Omega)$. Define, for each $s \in \mathbb{R}$, the real Hilbert space:

$$
\mathcal{H}^{s}:=\left\{f=\sum_{j=1}^{\infty} a_{j} e_{j}:\left(a_{j}\right)_{j \geq 1} \subset \mathbb{R}, \sum_{j=1}^{\infty}\left|a_{j}\right|^{2} \eta_{j}^{s}<+\infty\right\},
$$

equipped with the inner product:

$$
\left\langle f_{1}, f_{2}\right\rangle_{\mathcal{H}^{s}}:=\sum_{j=1}^{\infty} a_{j, 1} a_{j, 2} \eta_{j}^{s}, \quad f_{k}=\sum_{j=1}^{\infty} a_{j, k} e_{j} \in \mathcal{H}^{s}(k=1,2) .
$$


For all $t \geq 0, \Phi(t)$ belongs to $\mathcal{L}\left(\mathcal{H}^{s}\right)$ for any $s \in \mathbb{R}$ (see Proposition 7.1).

We now introduce the classes:

$$
\mathcal{H}^{-\infty}:=\bigcup_{s \in \mathbb{R}} \mathcal{H}^{s} \text { and } \mathcal{H}^{+\infty}:=\bigcap_{s \in \mathbb{R}} \mathcal{H}^{s}
$$

- Recall that for each continuous function $f$ over $\mathbb{R}^{+}$, the operator $f(-A)$ can be defined by the spectral functional calculus (see [15, Section 3, Chapter V.III]).

- Let us also define the following functions, related to the memory kernel $M$, that will play important roles in the decomposition of the flow.

First, we introduce the flow kernel:

$$
K_{M}(t, s):=\sum_{j=1}^{+\infty} \frac{(-s)^{j}}{j !} \underbrace{M * \cdots * M}_{j}(t-s), \quad(t, s) \in S_{+}:=\left\{(t, s) \in \mathbb{R}^{2}: t \geq s\right\} .
$$

Here and throughout the paper, $*$ denotes the usual convolution, i.e., when $g_{1}, g_{2} \in$ $L_{l o c}^{1}\left(\overline{\mathbb{R}^{+}}\right)$,

$$
g_{1} * g_{2}(t):=\int_{0}^{t} g_{1}(t-s) g_{2}(s) d s, t \geq 0 .
$$

Notice that the above $K_{M}$ is well-defined and it is real analytic over $S_{+}$(see Proposition 2.3). The following holds (see Proposition 4.8)

$$
\Phi(t)=e^{t A}+\int_{0}^{t} K_{M}(t, \tau) e^{\tau A} d \tau, \quad t \geq 0
$$

which yields a clear description of the gap between the heat equation and the memory one and justifies the terminology "flow kernel" employed.

Second, for each $N \in \mathbb{N}^{+}$, let

$$
R_{N}(t, \tau):=\int_{0}^{t} \tau e^{-\tau s} \partial_{s}^{N} K_{M}(t, s) d s, \quad t \geq 0, \quad \tau \geq 0 .
$$

Third, we define two sequences of functions $\left\{h_{l}\right\}_{l \in \mathbb{N}}$ and $\left\{p_{l}\right\}_{l \in \mathbb{N}}$ (that will play the role of coefficients in the expansions) in the following manner: for each $t \geq 0$,

$$
\left\{\begin{array}{l}
h_{l}(t):=(-1)^{l} \sum_{j=0}^{l} C_{l}^{l-j} \frac{d^{(l-j)}}{d t^{(l-j)}} \underbrace{M * \cdots * M}_{j}(t) ; \\
p_{l}(t):=-h_{l}(0)+(-1)^{l+1} \sum_{\substack{m, j \in \mathbb{N}^{+}, 2 j-l-1 \leq m \leq j}}^{M}(C_{l}^{l-j+m} \frac{d^{(l-j+m)}}{d t^{(l-j+m)}} \underbrace{M * \cdots * M}_{j}(0)) \frac{(-t)^{m}}{m !} .
\end{array}\right.
$$

Here, $C_{\beta}^{m}:=\beta ! / m !(\beta-m) !$ denotes the binomial coefficients and $\underbrace{M * \cdots * M}_{j}:=0$ when $j=0$. 
- Let $f$ be a distribution over a domain $\mathcal{D} \subset \mathbb{R}^{k}$ (with $k \in \mathbb{N}^{+}$). By the notation $f \in L_{\text {loc }}^{2}(q)$ (with $q \in \mathcal{D}$ ), we refer to the fact that $\left.f\right|_{\mathcal{U}_{0}} \in L^{2}\left(\mathcal{U}_{0}\right)$ for an open non-empty subset $\mathcal{U}_{0}$ such that $q \in \mathcal{U}_{0} \subset \mathcal{D}$.

The main results of this paper are as follows.

Theorem 1.1. For each integer $N \geq 2$, the flow $\Phi(t)$ admits the following decomposition:

$$
\Phi(t)=\mathcal{P}_{N}(t)+\mathcal{W}_{N}(t)+\mathfrak{R}_{N}(t), \quad t \geq 0
$$

with

$$
\left\{\begin{array}{ll}
\mathcal{P}_{N}(t) & :=e^{t A}+e^{t A} \sum_{l=0}^{N-1} p_{l}(t)(-A)^{-l-1}, \\
\mathcal{W}_{N}(t) & :=\sum_{l=0}^{N-1} h_{l}(t)(-A)^{-l-1}, \\
\mathfrak{R}_{N}(t) & :=R_{N}(t,-A)(-A)^{-N-1}
\end{array} \quad t \geq 0\right.
$$

where $\left\{h_{l}\right\}_{l \in \mathbb{N}}$ and $\left\{p_{l}\right\}_{l \in \mathbb{N}}$ are given by (1.8) and $R_{N}$ is given by (1.7). Moreover, for each $t \geq 0$, neither $\left\{h_{l}(t)\right\}_{l \geq 1}$ nor $\left\{p_{l}(t)\right\}_{l \in \mathbb{N}}$ is the null sequence, i.e.,

$$
\sum_{l \geq 1}\left|h_{l}(t)\right|>0 \quad \text { and } \quad \sum_{l \geq 1}\left|p_{l}(t)\right|>0 .
$$

Theorem 1.2. With the notation in Theorem 1.1, the following conclusions are true for each integer $N \geq 2$ :

(i) The first component $\mathcal{P}_{N}$ exhibits a heat-like behavior: for each $t>0, \mathcal{P}_{N}(t) \mathcal{H}^{-\infty} \subset \mathcal{H}^{+\infty}$, where $\mathcal{H}^{+\infty}$ and $\mathcal{H}^{-\infty}$ are given by $(1.5)$.

(ii) The second component $\mathcal{W}_{N}$ exhibits a wave-like behavior: when $y_{0} \in \mathcal{H}^{-\infty}, x_{0} \in \Omega$ and $t_{0}>0$,

$$
\mathcal{W}_{N}(\cdot) y_{0} \notin L_{l o c}^{2}\left(t_{0}, x_{0}\right) \Longleftrightarrow \forall t>0, \mathcal{W}_{N}(\cdot) y_{0} \notin L_{l o c}^{2}\left(t, x_{0}\right)
$$

In other words, the singularities of the solutions propagate in the time-like direction with null velocity of propagation in the space-like direction.

(iii) The last component $\mathfrak{R}_{N}$ exhibits a time-uniform smoothing effect with a gain of $2 N+2$ space derivatives: for each $y_{0} \in \mathcal{H}^{s}$ with $s \in \mathbb{R}, \mathfrak{R}_{N}(\cdot) y_{0} \in C\left([0,+\infty) ; \mathcal{H}^{s+2 N+2}\right)$, while $A^{-j} e^{\cdot A} y_{0}, A^{-j} y_{0} \in C\left([0,+\infty) ; \mathcal{H}^{s+2 j}\right)$ for any $0 \leq j \leq N$. And for each $s \in \mathbb{R}$, the term $R_{N}\left(\right.$ in $\left.\mathfrak{R}_{N}\right)$ belongs to $C\left(\mathbb{R}^{+} ; \mathcal{L}\left(\mathcal{H}^{s}\right)\right)$ and fulfills the estimate:

$$
\left\|R_{N}(t,-A)\right\|_{\mathcal{L}\left(\mathcal{H}^{s}\right)} \leq e^{t}\left\{\exp \left[N(1+t)\left(\sum_{j=0}^{N} \max _{0 \leq \tau \leq t}\left|\frac{d^{j}}{d \tau^{j}} M(\tau)\right|\right)\right]-1\right\}, \quad t \geq 0 .
$$

(iv) For any $y_{0} \in \mathcal{H}^{-\infty}, x_{0} \in \Omega$ and $t>0$,

$$
\Phi(\cdot) y_{0} \notin L_{l o c}^{2}\left(t, x_{0}\right) \Leftrightarrow \mathcal{W}_{N}(\cdot) y_{0} \notin L_{l o c}^{2}\left(t, x_{0}\right) \Leftrightarrow A^{-2} y_{0} \notin L_{l o c}^{2}\left(x_{0}\right) .
$$


Theorem 1.3. With the notation in Theorem 1.1, the following conclusions hold for each integer $N \geq 2$ : First, at the initial time,

$$
\lim _{j \rightarrow+\infty}\left\|\Phi(0) e_{j}\right\|_{L^{2}(\Omega)}=\lim _{j \rightarrow+\infty}\left\|\mathcal{P}_{N}(0) e_{j}\right\|_{L^{2}(\Omega)}=1, \lim _{j \rightarrow+\infty}\left\|\mathcal{W}_{N}(0) e_{j}\right\|_{L^{2}(\Omega)}=0, \mathfrak{R}_{N}(0)=0
$$

Second, at each time $t>0$,

$$
\left\{\begin{array}{l}
\lim _{j \rightarrow+\infty}\left\|\Phi(t) e_{j}\right\|_{\mathcal{H}^{4}}=\lim _{j \rightarrow+\infty}\left\|\mathcal{W}_{N}(t) e_{j}\right\|_{\mathcal{H}^{4}}=|M(t)|, \\
\lim _{j \rightarrow+\infty}\left\|\Phi(t) e_{j}\right\|_{\mathcal{H}^{s}}=\lim _{j \rightarrow+\infty}\left\|\mathcal{W}_{N}(t) e_{j}\right\|_{\mathcal{H}^{s}}=0 \text { for any } s<4 \\
\lim _{j \rightarrow+\infty}\left\|\mathcal{P}_{N}(t) e_{j}\right\|_{\mathcal{H}^{s}}=0 \text { for any } s \in \mathbb{R}, \\
\lim _{j \rightarrow+\infty}\left\|\Re_{N}(t) e_{j}\right\|_{\mathcal{H}^{s}}=0 \text { for any } s<2 N+2 .
\end{array}\right.
$$

Theorem 1.4. Given $s \in \mathbb{R}$, the following conclusions are true:

(i) There is $C_{0}>0$ (independent of $s$ ) so that for any $\alpha \in[0,4]$ and $t>0, \Phi(t)$ belongs to $\mathcal{L}\left(\mathcal{H}^{s}, \mathcal{H}^{s+\alpha}\right)$ and satisfies

$$
\|\Phi(t)\|_{\mathcal{L}\left(\mathcal{H}^{s}, \mathcal{H}^{s+\alpha}\right)} \leq C_{0} t^{-\frac{\alpha}{2}} \exp \left[2(1+t)\left(1+\|M\|_{C^{2}([0, t])}\right)\right] .
$$

(ii) If there is $\alpha_{0} \geq 0$ so that

$$
\Phi(t) \in \mathcal{L}\left(\mathcal{H}^{s}, \mathcal{H}^{s+\alpha_{0}}\right) \text { as } t>0 \text { in a neighborhood of } 0,
$$

then $\alpha_{0} \leq 4$ and $\liminf _{t \rightarrow 0^{+}} t^{\frac{\alpha_{0}}{2}}\|\Phi(t)\|_{\mathcal{L}\left(\mathcal{H}^{s}, \mathcal{H}^{\left.s+\alpha_{0}\right)}\right.}>0$.

(iii) For any $y_{0} \in \mathcal{H}^{s}, \Phi(\cdot) y_{0} \in C\left(\mathbb{R}^{+} ; \mathcal{H}^{s+4}\right)$. Moreover, the index 4 is optimal in the sense that if $\alpha>4$, then $\Phi(\cdot) \hat{y}_{0} \notin C\left(\mathbb{R}^{+} ; \mathcal{H}^{s+\alpha}\right)$ for some $\hat{y}_{0} \in \mathcal{H}^{s}$.

Remark 1.5. Several comments are in order:

(a1) Theorem 1.1 gives the decomposition (1.9) of the flow and besides shows the non-triviality of $\mathcal{P}_{N}$ and $\mathcal{W}_{N}$ : for each $t \geq 0$, there is $N_{0}(t) \in \mathbb{N}^{+}$so that $\mathcal{P}_{N}(t) \neq 0$ and $\mathcal{W}_{N}(t) \neq 0$ when $N \geq N_{0}(t)$. Theorem 1.2 explains the functionality of each term in the decomposition (1.9). Theorems 1.3-1.4 are the consequences of the decomposition (1.9). The three terms of the decomposition $\mathcal{P}_{N}, \mathcal{W}_{N}$ and $\mathfrak{R}_{N}$ are referred to as the heat-like component, the wave-like component and the remainder, respectively. The first two components are the leading ones. Due to their asymptotic expression, we can clearly identify their nature and this justifies the terminology heat/wave-like respectively. (This coincides with the expected hybrid nature of the flow.)

(a2) The proof of Theorem 1.1 uses a Fourier expansion on the basis of eigenfunctions of $A$ that reduces the problem to consider an ODE with memory depending on the dual parameter $\eta>0$ :

$$
w^{\prime}(t)+\eta w(t)+\int_{0}^{t} M(t-s) w(s) d s=0, \quad t>0 ; \quad w(0)=1 .
$$


The dynamics of this memory-ODE can be decomposed into three terms leading to the three components in the decomposition (1.9). The asymptotics of this decomposition for large $\eta$ yields the main properties of the decomposition (1.9) of the memory-heat equation. A careful analysis of the flow kernel $K_{M}$ plays a key role in this analysis.

(a3) In Theorem 1.2, the infinite order regularizing effect of the heat-like behavior of $\mathcal{P}_{N}$, stated in $(i)$, is the analog of the smoothing effect of the semigroup $\left\{e^{t A}\right\}_{t \geq 0}$ generated by the heat equation without memory terms; The wave-like component $\mathcal{W}_{N}$ exhibits the propagation of singularities along the time-direction, as stated in (ii); The smoothing effect of the remainder $\mathfrak{R}_{N}$, stated in (iii), ensures the gain of $2 N+2$ space-derivatives at nonnegative time but differs from the infinite order smoothing effect of the heat semigroup $e^{t A}$ at positive time (see Remark 4.4 for more discussions). The conclusion $(i v)$ says, in plain language, that when $t>0$, the singularity of $\mathcal{W}_{N}(t)$ determines the singularity of $\Phi(t)$, more precisely, the singularity of the practical leading term in $\mathcal{W}_{N}(t)$ determines the singularity of $\Phi(t)$. Here, we notice that it follows by (1.8) that $h_{0}(t) \equiv 0$ and $h_{1}(t)=$ $-M(t)$, thus the practical leading term in $\mathcal{W}_{N}(t)$ is $-M(t) A^{2}$ where the coefficient $M(t)$ is not zero except for finitely many $t$ by the assumption $(\mathfrak{C})$.

From these, we conclude that the decomposition (1.9) in Theorem 1.1 reveals the hybrid parabolic-hyperbolic behavior of the flow $\Phi(t)$.

(a4) Theorem 1.3 shows how the energy of solutions taking eigenfunctions of the operator $A$ as initial data is distributed over each component of (1.9) at time $t=0$ and time $t>0$ respectively. The conclusion (1.15) says that when $t=0$, the energy of $\Phi(0) e_{j}$ (which is exactly $\left.e_{j}\right)$ is almost concentrated in the heat-like component for large $j$, while (1.16) can be explained as: when $t>0$, the energy of $\Phi(t) e_{j}$ almost focuses on the wave-like component for large $j$. The first line in (1.16) is from the term $-M(t) A^{-2}$ (in $\mathcal{W}_{N}$ ) and the order 4 in $\mathcal{H}^{4}$ is exactly from the order -2 in $A^{-2}$. The last line in (1.16) is from (iii) in Theorem 1.2.

(a5) Theorem 1.4 exhibits the finite order smoothing effect of the flow. By it, we can see, from the point of view of the smoothing effect, both big differences and some similarities between the flow $\Phi(t)$ and the semigroup $e^{t A}$ :

First, on one hand, for any $y_{0} \in \mathcal{H}^{s}$ (with $\left.s \in \mathbb{R}\right), e^{\cdot A} y_{0} \in C\left(\mathbb{R}^{+} ; \mathcal{H}^{k}\right)(\forall k \in \mathbb{N})$, while $\Phi(\cdot) y_{0} \in C\left(\mathbb{R}^{+} ; \mathcal{H}^{s+4}\right)$ and moreover the index 4 is optimal, on the other hand, the smoothing effect of the flow $\Phi(t)$ at points in the set $\{t>0: M(t)=0\}$ is better than that at points in the set $\{t>0: M(t) \neq 0\}$ (see Remark 4.5 for more details);

Second, when $t$ is large, both the semigroup $e^{t A}$ and the flow $\Phi(t)$ are bounded from the above by exponential functions of $t$, while when $t$ is small, they are bounded from the above by the function $t^{-2}$. Moreover, the flow $\Phi(t)$ is also bounded from 
the below by $t^{-2}$ when $t \rightarrow 0^{+}$. Here, by " $e^{t A} / \Phi(t)$ is bounded by", we mean "the $\mathcal{L}\left(\mathcal{H}^{s}, \mathcal{H}^{s+4}\right)$-norm of $e^{t A} / \Phi(t)$ is bounded by".

(a6) It follows from Theorem 1.1 that for each $t \geq 0$, the wave-like component $\mathcal{W}_{N}(t)$, as well as the gap between the heat-like component $\mathcal{P}_{N}(t)$ and the heat semigroup $e^{t A}$, are nontrivial, when $N$ is large enough. Thus, we may expect that as $N$ increases the heat-like and the wave-like components include an increasing number of terms, just like in the Taylor expansion, and the decomposition becomes sharper. (See the example in Section 5.)

(a7) The last component $\mathfrak{R}_{N}$ does not fit completely into any of the two previous ones, but is needed in order to complete the representation of solutions. We further explain $\mathfrak{R}_{N}$ as follows:

- Hybridity. It inherits a hybrid heat/wave structure from the flow (see the note (R2) in Remark 5.2).

- Smoothing effect. It has the time-uniform smoothing effect given in (iii) of Theorem 1.2. Such time-uniform smoothing effect differs from the usual smoothing effect of the heat semigroup. (This has be mentioned in (a3)).

From Theorems 1.1-1.2, as well as the example in Section 5, we can see what follows: First, when $t=0, \mathfrak{R}_{N}(t)$ has a better smoothing effect than that of $\mathcal{P}_{N}(t)$, but when $t>0$, this is reversed. (The reason why the smoothing effect of $\mathfrak{R}_{N}(t)$ (with $t>0$ ) is weaker than that of $\mathcal{P}_{N}(t)$ is due to wave-like terms contained in $\mathfrak{R}_{N}(t)$.) Second, when $t>0$, both $\mathfrak{R}_{N}(t)$ and $\mathcal{P}_{N}(t)$ have better smoothing effects than that of $\mathcal{W}_{N}(t)$, thus as $t>0$, the singularity of the flow is dominated by the wave-like component and the flow shows its wave-like nature.

- Frequencies. In Fourier analysis, the smoothing effect of a pseudo-differential operator corresponds to the growth of its symbol at high frequencies. The situation here is similar-Theorem 1.1 and Theorem 3.1 corresponds to each other and the smoothing effects of the components in (1.9) correspond to respectively the growths (in large $\eta$ ) of the components in (3.2). From this and (3.2), we can see that when $t=0$, the last component in (3.2) (It corresponds to $\mathfrak{R}_{N}$.) has a faster decay (in large $\eta$ ) than the first two components in (3.2), while when $t>0$, both the last one and the first one have faster decay than the second one.

- Non-triviality. Since the component $\mathfrak{R}_{N}$ has a hybrid heat/wave structure as mentioned above (In particular, we would like to mention that it is not a finite dimensional low frequency operator.) it is not negligible.

(a8) We require $N \geq 2$ in Theorem 1.1 since $\mathcal{W}_{1}(\cdot) \equiv 0($ when $N=1)$. 
(a9) Notice that both $\mathcal{P}_{N}(0)$ and $\mathcal{W}_{N}(0)$ are not projection operators in general and that

$$
y_{0}=\mathcal{P}_{N}(0) y_{0}+\mathcal{W}_{N}(0) y_{0} \text { for each } y_{0} \in L^{2}(\Omega) \text { and } \mathfrak{R}_{N}(0)=0
$$

This is further discussed in Proposition 4.7.

(a10) All the results of Theorems 1.1-1.2, except (1.11), (1.12) and (1.14), hold under the weaker assumption $M \in C^{\infty}\left(\overline{\mathbb{R}^{+}}\right)$. In Theorem 4.10 below we analyse the case of kernels $M$ in $C^{N_{0}}\left(\overline{\mathbb{R}^{+}}\right)$, with $N_{0} \geq 2$. The assumption ( $\mathfrak{C}$ ) ensures (1.11), (1.12) and (1.14). Whether the same holds under weaker conditions on the kernel is an open problem, see Section 6 .

(a11) The decomposition (1.9) has applications in control theory. It allows, in particular, to compare the reachable sets for the controlled heat equations with and without memory term. We refer to [21] for a complete analysis of this issue.

(a12) There is a large body of literature on the large time dynamics of memory like problems (see, for instance, $[8,9]$ ) which is surely an important direction. Unfortunately, we are not able to use our decomposition to get such results.

\subsection{Plan of the paper}

The rest of the paper is organized as follows. In Section 2 we analyze the flow kernel $K_{M}$. In Section 3 we present a decomposition for solutions to the ODE (1.19). Section 4 contains the proofs of Theorems 1.1-1.4, and provides some other properties of the flow. In Section 5 we discuss, as an example, the case of the kernel $M(t)=\alpha e^{\lambda t}$. Section 6 lists several open problems. Section 7 contains an appendix.

\section{Properties of the flow kernel}

In this section we present some properties of the flow kernel $K_{M}$ in (1.6), which will be used later.

In what follows, the space $C^{k}([a, b])$ (with $k \in \mathbb{N}^{+}$and $\left.a<b\right)$ is endowed with the norm:

$$
\|f\|_{C^{k}([a, b])}:=\sum_{l=0}^{k}\left\|\frac{d^{l} f}{d x^{l}}\right\|_{C([a, b])}, \quad f \in C^{k}([a, b]) .
$$

The following result provides basic estimates on iterated convolutions that will be used in the proof of Proposition 2.2. Its proof is put in the appendix.

Lemma 2.1. Let $j, m \in \mathbb{N}^{+}$. Then for each sequence $\left\{M_{l}\right\}_{l=1}^{j} \subset C^{m}\left(\overline{\mathbb{R}^{+}}\right), M_{1} * \cdots * M_{j}$ is in the space $C^{m+j-1}\left(\overline{\mathbb{R}^{+}}\right)$and satisfies that for each $k \in\{0,1, \ldots, m+j-1\}$,

$$
\left|\frac{d^{k}}{d t^{k}} M_{1} * \cdots * M_{j}(t)\right| \leq\left(\sum_{l=\max \{0, j-1-k\}}^{j-1} \frac{t^{l}}{l !}\right) \prod_{l=1}^{j}\left\|M_{l}\right\|_{C^{p}([0, t])}, \quad t>0,
$$

where $p:=\chi_{\mathbb{N}}(k-1)\left[(k-j) \chi_{\mathbb{N}}(k-j)+1\right]$ and $\chi_{\mathbb{N}}$ is the characteristic function of the set $\mathbb{N}$. 
The following Proposition 2.2 provides estimates on the derivatives of the flow kernel $K_{M}$ that will serve for the proof of Theorem 3.1, which is one of the tools in the proof of the decomposition in Theorem 1.1.

Proposition 2.2. The flow kernel $K_{M} \in C^{\infty}\left(S_{+}\right)$(where $S_{+}$is given in (1.6)) satisfies that for each $\alpha, \beta \in \mathbb{N}$,

$$
\left|\partial_{t}^{\alpha} \partial_{s}^{\beta} K_{M}(t, s)\right| \leq e^{t-s}\left[\exp \left(\beta(1+|s|)\|M\|_{C^{\alpha+\beta}([0, t-s])}\right)-1\right], \quad t>s .
$$

Proof. First of all, it follows from (1.6) that

$$
K_{M}(t, s)=\sum_{j=1}^{+\infty} \mathcal{M}_{j}(t, s), \quad t \geq s
$$

where

$$
\mathcal{M}_{j}(t, s):=\frac{(-s)^{j}}{j !} \underbrace{M * \cdots * M}_{j}(t-s), \quad t \geq s .
$$

Next, we prove $K_{M} \in C^{\infty}\left(S_{+}\right)$showing the convergence in $C^{\infty}\left(S_{+}\right)$of the series on the right-hand side of (2.3).

To this end, we will estimate $\mathcal{M}_{j}$ with $j \in \mathbb{N}^{+}$: By the assumption ( $\mathfrak{C}$ ) and Lemma 2.1, we see that $\underbrace{M * \cdots * M}_{j}$ belongs to $C^{\infty}\left(\overline{\mathbb{R}^{+}}\right)$and satisfies, for each $k \in \mathbb{N}$,

$$
|\frac{d^{k}}{d \tau^{k}} \underbrace{M * \cdots * M}_{j}(\tau)| \leq e^{\tau}\|M\|_{C^{k}([0, \tau])}^{j}, \quad \tau>0 .
$$

From (2.4) it follows that $\mathcal{M}_{j} \in C^{\infty}\left(S_{+}\right)$.

By direct computations, for $\alpha, \beta \in \mathbb{N}$, we have $t \geq s$,

$$
\begin{aligned}
\partial_{t}^{\alpha} \partial_{s}^{\beta} \mathcal{M}_{j}(t, s) & =\partial_{s}^{\beta} \partial_{t}^{\alpha} \mathcal{M}_{j}(t, s) \\
& =\frac{d^{\beta}}{d s^{\beta}}\left[\left.\frac{(-s)^{j}}{j !}(\frac{d^{\alpha}}{d \tau^{\alpha}} \underbrace{M * \cdots * M}_{j}(\tau))\right|_{\tau=t-s}\right] \\
& =\left.\sum_{m=0}^{\beta} C_{\beta}^{m} \frac{d^{m}}{d \tau^{m}}\left(\frac{(-s)^{j}}{j !}\right)(-1)^{\beta-m}(\frac{d^{\beta-m}}{d \tau^{\beta-m}} \frac{d^{\alpha}}{d \tau^{\alpha}} \underbrace{M * \cdots * M}_{j}(\tau))\right|_{\tau=t-s} \\
& =\left.\frac{(-1)^{\beta}}{j !} \sum_{m=0}^{\min \{\beta, j\}} C_{\beta}^{m}\left(C_{j}^{m} m !\right)(-s)^{j-m}(\frac{d^{\alpha+\beta-m}}{d \tau^{\alpha+\beta-m}} \underbrace{M * \cdots * M}_{j}(\tau))\right|_{\tau=t-s} .
\end{aligned}
$$

Here and in what follows, we use the conventional notation $0^{0}:=1$. By (2.6) and (2.5), one has 
that, when $t>s$,

$$
\begin{aligned}
\left|\partial_{t}^{\alpha} \partial_{s}^{\beta} \mathcal{M}_{j}(t, s)\right| & \leq \frac{1}{j !} \sum_{m=0}^{j}\left(C_{\beta}^{m} m !\right) C_{j}^{m}|s|^{j-m}\left(e^{t-s}\|M\|_{C^{\alpha+\beta}([0, t-s])}^{j}\right) \\
& \leq \frac{1}{j !} \beta^{j}\left(\sum_{m=0}^{j} C_{j}^{m}|s|^{j-m}\right) e^{t-s}\|M\|_{C^{\alpha+\beta}([0, t-s])}^{j} \\
& =\frac{e^{t-s}}{j !}\left(\beta(1+|s|)\|M\|_{C^{\alpha+\beta}([0, t-s])}\right)^{j} .
\end{aligned}
$$

Now, by (2.7) it follows that the series in (2.3) converges in $C^{\infty}\left(S_{+}\right)$.

Finally, by (2.3) and (2.7), after direct computations, we see that when $t>s$,

$$
\left|\partial_{t}^{\alpha} \partial_{s}^{\beta} K_{M}(t, s)\right| \leq \sum_{j=1}^{\infty}\left|\partial_{t}^{\alpha} \partial_{s}^{\beta} \mathcal{M}_{j}(t, s)\right| \leq e^{t-s}\left[\exp \left(\beta(1+|s|)\|M\|_{C^{\alpha+\beta}([0, t-s])}\right)-1\right] .
$$

This gives the desired estimate (2.2) and ends the proof of Proposition 2.2.

The next Proposition 2.3 concerns the analyticity of the flow kernel $K_{M}$. It will be used in the proofs of Proposition 2.4 and Proposition 4.9.

Proposition 2.3. The flow kernel $K_{M}$ is real analytic on $S_{+}$(where $S_{+}$is given in (1.6)).

Proof. It suffices to prove that $K_{M}$ is real analytic over $S_{T}$ for each $T>0$, where

$$
S_{T}:=\left\{(t, s) \in \mathbb{R}^{2}: 0 \leq t-s \leq T\right\}
$$

To this end, we fix an arbitrary $T>0$. Due to the analyticity of $M$ over $\overline{\mathbb{R}^{+}}$, there is a domain $\widetilde{\mathcal{O}_{T}}$ of the complex plane $\mathbb{C}$, with $[0, T] \subset \widetilde{\mathcal{O}_{T}} \subset \mathbb{C}$, so that $M$ has a unique analytic extension $\widetilde{M}$ to $\widetilde{\mathcal{O}_{T}}$. Moreover, we can take a bounded and convex subdomain $\mathcal{O}_{T}$ so that $[0, T] \subset \mathcal{O}_{T} \subset \subset \widetilde{\mathcal{O}_{T}}$. The convolution $*$ can then be extended to $\tilde{*}$ in the following manner for $f, g \in C\left(\mathcal{O}_{T} ; \mathbb{C}\right)$,

$$
f \tilde{*} g(z):=\int_{0}^{1} f((1-s) z) g(s z) z d s, z \in \mathcal{O}_{T} .
$$

We now claim the following two properties:

(P1) For each $j \in \mathbb{N}^{+}, \underbrace{\widetilde{M} \widetilde{*} \tilde{*} \widetilde{M}}_{j}$ is an analytic extension of $\underbrace{M * \cdots * M}_{j}$ over $\mathcal{O}_{T}$;

(P2) There is $C>0$ so that

$$
\sup _{z \in \mathcal{O}_{T}}|\underbrace{\widetilde{M} \tilde{*} \cdots \tilde{*} \widetilde{M}}_{j}(z)| \leq C^{j} \text { for all } j \in \mathbb{N}^{+} .
$$

Indeed, one has

$$
\left.f \tilde{*}\right|_{[0, T]}=\left.\left.f\right|_{[0, T]} * g\right|_{[0, T]}, \quad \text { when } f, g \in C\left(\mathcal{O}_{T} ; \mathbb{C}\right),
$$


Here, $\left.f \tilde{*} g\right|_{[0, T]},\left.f\right|_{[0, T]}$ and $\left.g\right|_{[0, T]}$ are respectively the restrictions of $f \tilde{*} g, f$ and $g$ over $[0, T]$. Then, property (P1) follows from the analyticity of $M$ at once, while the property (P2) can be proved by direct computations.

Define the following subset of $\mathbb{C}^{2}$ :

$$
\mathcal{D}_{T}:=\left\{(t, s) \in \mathbb{C}^{2}: t-s \in \mathcal{O}_{T}\right\} .
$$

It is clear that $S_{T} \subset \mathcal{D}_{T}$. According to (P2) above, the following series uniformly converges over each compact subset of $\mathcal{D}_{T}$ :

$$
\sum_{j \geq 1} \frac{(-s)^{j}}{j !} \underbrace{\widetilde{M} \tilde{*} \cdots \widetilde{*}}_{j}(t-s), \quad(t, s) \in \mathcal{D}_{T} .
$$

Meanwhile, by (P1), we find that each term in the above series is analytic over $\mathcal{D}_{T}$. Hence, the sum of this series is analytic over $\mathcal{D}_{T}$. From this and (1.6), we see that $\left.K_{M}\right|_{S_{T}}$ (the restriction of $K_{M}$ over $S_{T}$ ) can be analytically extended to $\mathcal{D}_{T}$. Therefore, it is real analytic over $S_{T}$. This ends the proof of Proposition 2.3.

The next Proposition 2.4 can be interpreted as a strong unique continuation property or non-degeneracy of the kernel $K_{M}$, that will be used in the proof of Theorem 3.1.

Proposition 2.4. For each $\left(t_{0}, s_{0}, v_{1}, v_{2}\right) \in\left(S_{+} \times \mathbb{S}^{1}\right) \backslash I$, where

$$
I:=\{(t, t, \tau, \tau): t \in \mathbb{R}, \tau= \pm 1 / \sqrt{2}\} \cup\{(t, 0, \tau, 0): t \geq 0, \tau= \pm 1\},
$$

with $\partial_{\vec{v}}:=v_{1} \partial_{t}+v_{2} \partial_{s}$ where $\vec{v}:=\left(v_{1}, v_{2}\right)$, it holds that

$$
\partial_{\vec{v}}^{l} K_{M}\left(t_{0}, s_{0}\right) \neq 0 \text { for some } l \in \mathbb{N} \text {. }
$$

Remark 2.5. Obviously, real analytic functions on $\mathbb{R}^{2}$ do not necessarily fulfill the non-degeneracy condition above. Indeed, polynomials, for instance, can vanish along lines in $\mathbb{R}^{2}$.

Proof of Proposition 2.4. It suffices to prove $(2.8)$ in the case that $\vec{v}=(0,1)$, as other cases can be proved in a very similar way. By contradiction, suppose that $(2.8)$ with $\vec{v}=(0,1)$ fails, i.e.,

$$
\partial_{(0,1)}^{l} K_{M}\left(t_{0}, s_{0}\right)=0 \text { for all } l \in \mathbb{N} .
$$

Define

$$
f(\lambda):=K_{M}\left(t_{0}, t_{0}-\lambda\right), \quad \lambda \geq 0 .
$$

Two facts on $f$ are given as follows: First, by the real analyticity of $M$ over $\overline{\mathbb{R}^{+}}$, we see from Proposition 2.3 that $K_{M}$ is real analytic over $S_{+}$. This, along with (2.10), yields that $f$ is real analytic over $\overline{\mathbb{R}^{+}}$. Second, by $(2.9)$ and (2.10), we find that $f$ vanishes of infinite order at $\lambda=t_{0}-s_{0}$. From these two facts, we see that $f \equiv 0$ over $\overline{\mathbb{R}^{+}}$, which, along with (2.10), yields

$$
K_{M}\left(t_{0}, t_{0}-\lambda\right)=0, \quad \lambda \geq 0 .
$$


The above, together with (1.6), shows

$$
0=\sum_{j=1}^{+\infty} \frac{\left(\lambda-t_{0}\right)^{j}}{j !} \underbrace{M * \cdots * M}_{j}(\lambda), \quad \lambda \geq 0,
$$

which leads to

$$
0=M(\lambda)+\sum_{j=2}^{+\infty} \frac{\left(\lambda-t_{0}\right)^{j-1}}{j !} \underbrace{M * \cdots * M}_{j}(\lambda), \quad \lambda \geq 0 .
$$

Next, we arbitrarily fix $T>0$. For each $j \in \mathbb{N}^{+} \backslash\{1\}$, we define an operator $\mathcal{K}_{j}$ on $C([0, T])$ in the following manner: given $g \in C([0, T])$, set

$$
\mathcal{K}_{j}(g)(\lambda):=\frac{\left(\lambda-t_{0}\right)^{j-1}}{j !} \underbrace{M * \cdots * M}_{j-1} * g(\lambda), \quad 0 \leq \lambda \leq T,
$$

which is well-defined, linear and bounded. Let

$$
\mathcal{Q}:=\sum_{j=2}^{+\infty} \mathcal{K}_{j}
$$

One can directly check that $\mathcal{Q} \in \mathcal{L}(C([0, T]))$, the Banach space of all linear and bounded operators on $C([0, T])$. Thus, we deduce from (2.11) that

$$
(I d+\mathcal{Q})\left(\left.M\right|_{[0, T]}\right)=\left(I d+\sum_{j=2}^{+\infty} \mathcal{K}_{j}\right)\left(\left.M\right|_{[0, T]}\right)=0,
$$

where $I d$ is the identity operator on $C([0, T])$.

We now claim that $(I d+\mathcal{Q})^{-1}$ exists in $\mathcal{L}(C([0, T]))$. When this is done, we can use $(2.13)$, (1.6) and the arbitrariness of $T$ to see that $M \equiv 0$, which contradicts the assumption ( $\mathfrak{C}$ ). Consequently, (2.8) is true.

The remainder is to show the above claim. To this end, we arbitrarily fix $k \in \mathbb{N}^{+}$and then estimate $\mathcal{Q}^{k}$ in the following manner: Set

$$
T_{t_{0}}:=\sup _{0 \leq \lambda \leq T}\left|\lambda-t_{0}\right|=\max \left\{\left|t_{0}\right|,\left|T-t_{0}\right|\right\} .
$$

Then from (2.12), one can directly check that when $j_{1}, \ldots, j_{k} \geq 2$,

$$
\begin{aligned}
\left\|\mathcal{K}_{j_{1}} \cdots \mathcal{K}_{j_{k}}\right\|_{\mathcal{L}(C([0, T]))} & \leq \frac{1}{j_{1} !} \cdots \frac{1}{j_{k} !} T_{t_{0}}^{j_{1}+\cdots+j_{k}-k}\|\underbrace{|M| * \cdots *|M|}_{j_{1}+\cdots+j_{k}-k}\|_{L^{1}([0, T])} \\
& \leq \frac{1}{j_{1} !} \cdots \frac{1}{j_{k} !} T_{t_{0}}^{j_{1}+\cdots+j_{k}-k} \frac{T^{j_{1}+\cdots+j_{k}-k}}{\left(j_{1}+\cdots+j_{k}-k\right) !}\|M\|_{C([0, T])}^{j_{1}+\cdots+j_{k}-k} \\
& \leq \frac{1}{k !} \frac{1}{j_{1} !} \cdots \frac{1}{j_{k} !}\left(T_{t_{0}} T\|M\|_{C([0, T])}\right)^{j_{1}+\cdots+j_{k}-k}
\end{aligned}
$$


This, along with the definition of $\mathcal{Q}$, yields

$$
\begin{gathered}
\left\|\mathcal{Q}^{k}\right\|_{\mathcal{L}(C([0, T]))}=\left\|\sum_{j_{1}, \ldots, j_{k} \geq 2} \mathcal{K}_{j_{1}} \cdots \mathcal{K}_{j_{k}}\right\|_{\mathcal{L}(C([0, T]))} \\
\leq \frac{1}{k !} \prod_{m=1}^{k}\left(\sum_{j_{m} \geq 2} \frac{1}{j_{m} !}\left(T_{t_{0}} T\|M\|_{C([0, T])}\right)^{j_{m}-1}\right) \leq \frac{1}{k !} \exp \left(k T_{t_{0}} T\|M\|_{C([0, T])}\right) .
\end{gathered}
$$

So $\sum_{k=0}^{+\infty}(-\mathcal{Q})^{k}$ converges in $\mathcal{L}(C([0, T]))$. Then we have

$$
I d=(I d+\mathcal{Q}) \sum_{k=0}^{+\infty}(-\mathcal{Q})^{k} .
$$

Therefore, $(I d+\mathcal{Q})^{-1}$ exists in $\mathcal{L}(C([0, T]))$. This completes the proof of Proposition 2.4.

The following Proposition 2.6 presents a weighted estimate of the flow kernel $K_{M}$. It will be used in the proof of Proposition 4.8 that provides an explicit expression of the gap between the heat evolution with and without memory.

Proposition 2.6. For each $\lambda \in \mathbb{R}$,

$$
\int_{0}^{t} e^{-\lambda(t-s)}\left|K_{M}(t, s)\right| d s \leq \exp \left(t \int_{0}^{t} e^{-\lambda \tau}|M(\tau)| d \tau\right)-1, \quad t \geq 0 .
$$

Proof. Arbitrarily fix $\lambda \in \mathbb{R}$. Define the following weighted memory kernel:

$$
M_{\lambda}(t):=e^{-\lambda t} M(t), \quad t \geq 0 .
$$

We claim

$$
e^{-\lambda(t-s)} K_{M}(t, s)=\sum_{j=1}^{+\infty} \frac{(-s)^{j}}{j !} \underbrace{M_{\lambda} * \cdots * M_{\lambda}}_{j}(t-s), \quad(t, s) \in S_{+} .
$$

Indeed, (2.16) follows from (1.6) and the following identity:

$$
\underbrace{M_{\lambda} * \cdots * M_{\lambda}}_{j}(\tau)=e^{-\lambda \tau} \underbrace{M * \cdots * M}_{j}(\tau), \quad \tau \geq 0, j \in \mathbb{N}^{+},
$$

which can be verified directly.

Next, we arbitrarily fix $t>0$. By the iterative use of the Young's inequality:

$$
\|f * g\|_{L^{1}(0, t)} \leq\|f\|_{L^{1}(0, t)}\|g\|_{L^{1}(0, t)} \text {, when } f, g \in L^{1}(0, t),
$$

one has that

$$
\|\underbrace{M_{\lambda} * \cdots * M_{\lambda}}_{j}\|_{L^{1}(0, t)} \leq\left\|M_{\lambda}\right\|_{L^{1}(0, t)}^{j}, \quad j \in \mathbb{N}^{+} .
$$

This, along with (2.16), yields

$$
\int_{0}^{t}\left|e^{-\lambda(t-s)} K_{M}(t, s)\right| d s \leq \sum_{j=1}^{+\infty} \frac{t^{j}}{j !}\|\underbrace{M_{\lambda} * \cdots * M_{\lambda}}_{j}\|_{L^{1}(0, t)} \leq \exp \left(t\left\|M_{\lambda}\right\|_{L^{1}(0, t)}\right)-1 .
$$

Then (2.14) follows from (2.15). This concludes the proof of Proposition 2.6. 


\section{Parameterized ODEs with memory}

In this section we analyze the ODE (1.19), i.e.,

$$
w^{\prime}(t)+\eta w(t)+\int_{0}^{t} M(t-s) w(s) d s=0, \quad t>0 ; \quad w(0)=1,
$$

where $\eta>0$ is a parameter. First of all, by a standard method in the ODE theory, one can easily check that the equation (3.1) has a unique solution, denoted by $w_{\eta}$, in the space $C^{1}\left(\overline{\mathbb{R}^{+}}\right)$. The main result of this section is the next Theorem 3.1 which gives a decomposition in terms of $\eta$ for the solution $w_{\eta}$. It lays a solid foundation for the proof of Theorem 1.1.

Theorem 3.1. For each integer $N \geq 2$, the solution $w_{\eta}$ (with $\eta>0$ ) to the equation (3.1) satisfies

$$
w_{\eta}(t)=e^{-\eta t}\left(1+\sum_{l=0}^{N-1} p_{l}(t) \eta^{-l-1}\right)+\sum_{l=0}^{N-1} h_{l}(t) \eta^{-l-1}+R_{N}(t, \eta) \eta^{-N-1}, \quad t \geq 0,
$$

where $\left\{h_{l}\right\}_{l \in \mathbb{N}}$ and $\left\{p_{l}\right\}_{l \in \mathbb{N}}$ are given by (1.8) and $R_{N}$ is given by (1.7). In addition, the following conclusions are true:

(i) The function $R_{N}$ is in the space $C\left(\overline{\mathbb{R}^{+}} \times \overline{\mathbb{R}^{+}}\right) \cap C\left(\mathbb{R}^{+} ; C\left(\mathbb{R}^{+}\right)\right)$and fulfills the estimate:

$$
\left\|R_{N}(t, \cdot)\right\|_{C\left(\mathbb{R}^{+}\right)} \leq e^{t}\left\{\exp \left[N(1+t)\left(\sum_{j=0}^{N} \max _{0 \leq s \leq t}\left|\frac{d^{j}}{d s^{j}} M(s)\right|\right)\right]-1\right\}, \quad t \geq 0 .
$$

(ii) For each $t \in \overline{\mathbb{R}^{+}}$, neither $\left\{h_{l}(t)\right\}_{l \geq 1}$ nor $\left\{p_{l}(t)\right\}_{l \in \mathbb{N}}$ is the null sequence.

Remark 3.2. (i) The decomposition (3.2) serves for that in (1.9). The components in the decompositions (3.2) and (1.9) correspond to each other.

(ii) The conclusion (ii) in Theorem 3.1 corresponds to (1.11) in Theorem 1.1 discussed in Remark 1.5.

To prove Theorem 3.1, we need several lemmas. The first one refers to the representation of the solutions of (3.1) in terms of the flow kernel $K_{M}$ (given by (1.6)).

Lemma 3.3. The solution $w_{\eta}$ (with $\eta \in \mathbb{R}$ ) to (3.1) satisfies

$$
w_{\eta}(t)=e^{-\eta t}+\int_{0}^{t} K_{M}(t, s) e^{-\eta s} d s, \quad t \geq 0 .
$$

Proof. Fix an arbitrary $\eta \in \mathbb{R}$. From (3.1), it follows that

$$
w_{\eta}^{\prime}(t)+\eta w_{\eta}(t)=-M * w_{\eta}(t), t \geq 0 ; \quad w_{\eta}(0)=1 .
$$

The above yields

$$
w_{\eta}(t)=e^{-\eta t}-\int_{0}^{t} e^{-\eta(t-s)}\left(M * w_{\eta}\right)(s) d s=e^{-\eta t}-\left(e^{-\eta \cdot} * M * w_{\eta}\right)(t), t \geq 0 .
$$


Then we arbitrarily fix $T>0$ and define the following operator:

$$
\mathcal{Q}_{T}(f):=e^{-\eta \cdot} * M * f \text { for each } f \in C([0, T])
$$

One can easily check that $\mathcal{Q}_{T}$ is a linear and bounded operator on $C([0, T])$. By (3.6), we see that for each $k \in \mathbb{N}^{+}$,

$$
\mathcal{Q}_{T}^{k}(f)=\underbrace{\left(e^{-\eta \cdot} * M\right) * \cdots *\left(e^{-\eta \cdot} * M\right)}_{k} * f, \quad f \in C([0, T]) .
$$

From (3.7), one can directly check

$$
\begin{aligned}
\left\|\mathcal{Q}_{T}^{k}\right\|_{\mathcal{L}(C([0, T]))} & \leq\|\underbrace{\left(e^{-\eta \cdot} * M\right) * \cdots *\left(e^{-\eta \cdot} * M\right)}_{k}\|_{L^{1}(0, T)} \\
& \leq\left\|e^{-\eta \cdot} * M\right\|_{C([0, T])}^{k}\left(\int_{0}^{T} \int_{0}^{t_{1}} \cdots \int_{0}^{t_{k-1}} d t_{k} \cdots d t_{1}\right) \\
& \leq\left(e^{T|\eta|}\|M\|_{L^{1}(0, T)}\right)^{k} \frac{T^{k}}{k !} .
\end{aligned}
$$

From this, we see that the series $\sum_{k=0}^{\infty}\left(-\mathcal{Q}_{T}\right)^{k}$ converges in $\mathcal{L}(C([0, T]))$ and that

$$
\left(1+\mathcal{Q}_{T}\right)^{-1}=\sum_{k=0}^{\infty}\left(-\mathcal{Q}_{T}\right)^{k}
$$

Meanwhile, it follows from (3.5) and (3.6) that

$$
\left.w_{\eta}\right|_{[0, T]}=-\mathcal{Q}_{T}\left(\left.w_{\eta}\right|_{[0, T]}\right)+e^{-\left.\eta \cdot\right|_{[0, T]}} \cdot
$$

Here, $\left.w_{\eta}\right|_{[0, T]}$ and $\left.e^{-\eta \cdot}\right|_{[0, T]}$ denote respectively the restrictions of $w_{\eta}$ and $e^{-\eta \cdot}$ over $[0, T]$.

Now, by (3.9) and (3.8), we find

$$
w_{\eta}(t)=\left(\left(\sum_{j=0}^{\infty}\left(-\mathcal{Q}_{T}\right)^{j}\right)\left(\left.e^{-\eta \cdot}\right|_{[0, T]}\right)\right)(t), t \in[0, T]
$$

from which and (3.7), it follows that

$$
\begin{aligned}
w_{\eta}(t) & =e^{-\eta t}+\sum_{j=1}^{\infty}(-1)^{j} \underbrace{\left(e^{-\eta \cdot} * M\right) * \cdots *\left(e^{-\eta \cdot} * M\right)}_{j} * e^{-\eta \cdot}(t) \\
& =e^{-\eta t}+\sum_{j=1}^{\infty}(-1)^{j} \underbrace{e^{-\eta \cdot} * \cdots * e^{-\eta \cdot}}_{j+1} * \underbrace{M * \cdots * M}_{j}(t), t \in[0, T] .
\end{aligned}
$$

This, together with the following equality:

$$
\underbrace{e^{-\eta \cdot} * \cdots * e^{-\eta \cdot}}_{j+1}(t)=e^{-\eta t} t^{j} / j !, \quad t \geq 0, \quad j \in \mathbb{N}^{+},
$$


shows

$$
w_{\eta}(t)=e^{-\eta t}+\sum_{j=1}^{\infty} \int_{0}^{t} e^{-\eta s} \frac{(-s)^{j}}{j !} \underbrace{M * \cdots * M}_{j}(t-s) d s, t \in[0, T] .
$$

Since $T>0$ was arbitrarily taken, the above, along with (1.6), leads to (3.4). This ends the proof of Lemma 3.3.

The next lemma will be used to get an asymptotic expansion of the second term on the right-hand side of (3.4).

Lemma 3.4. Given an integer $N \geq 2$ and a number $\eta>0$, the following equality is true:

$$
\begin{gathered}
\int_{0}^{t} e^{-\eta s} K_{M}(t, s) d s=e^{-\eta t} \sum_{l=0}^{N-1}\left(-\left.\partial_{s}^{l} K_{M}(t, s)\right|_{s=t}\right) \eta^{-l-1}+\sum_{l=0}^{N-1}\left(\left.\partial_{s}^{l} K_{M}(t, s)\right|_{s=0}\right) \eta^{-l-1} \\
+\eta^{-N} \int_{0}^{t} e^{-\eta s} \partial_{s}^{N} K_{M}(t, s) d s, \quad t \geq 0
\end{gathered}
$$

Proof. Fix $N \geq 2, \eta>0$ and $t>0$ arbitrarily. Given $g \in C([0, t])$, we define

$$
\mathcal{F}_{\eta}(g):=\int_{0}^{t} e^{-\eta s} g(s) d s
$$

We first claim

$$
\mathcal{F}_{\eta}(g)=\eta^{-N} \mathcal{F}_{\eta}\left(g^{(N)}\right)+\sum_{l=0}^{N-1} \eta^{-l-1}\left(g^{(l)}(0)-e^{-\eta t} g^{(l)}(t)\right), \text { when } g \in C^{N}([0, t]) .
$$

Given $g \in C^{N}([0, t])$, from (3.11) and using the integration by parts, we find

$$
\begin{aligned}
\mathcal{F}_{\eta}(g) & =(-\eta)^{-1} \int_{0}^{t} \frac{d}{d s} e^{-\eta s} g(s) d s \\
& =\left.(-\eta)^{-1}\left(e^{-\eta s} g(s)\right)\right|_{s=0} ^{t}+\frac{1}{\eta} \int_{0}^{t} e^{-\eta s} g^{\prime}(s) d s \\
& =\eta^{-1}\left(g(0)-e^{-\eta t} g(t)\right)+\eta^{-1} \mathcal{F}_{\eta}\left(g^{\prime}\right) .
\end{aligned}
$$

Now, the iterative use of the above equality (to the derivatives of $g$ ) gives (3.12).

Next, it follows by Proposition 2.2 that $K_{M}(t, \cdot) \in C^{\infty}([0, t])$. Thus we can apply (3.12) (where $g(s)=K_{M}(t, s), 0 \leq s \leq t$ ) to get

$$
\begin{aligned}
\int_{0}^{t} K_{M}(t, s) e^{-\eta s} d s= & \eta^{-N} \int_{0}^{t} e^{-\eta s} \partial_{s}^{N} K_{M}(t, s) d s \\
& +\sum_{l=0}^{N-1} \eta^{-l-1}\left(\left.\partial_{s}^{l} K_{M}(t, s)\right|_{s=0}-\left.e^{-\eta t} \partial_{s}^{l} K_{M}(t, s)\right|_{s=t}\right) .
\end{aligned}
$$

This leads to (3.10) and completes the proof of Lemma 3.4.

The following Lemma 3.5 will be used in the proof of $(i i)$ in Theorem 3.1. 
Lemma 3.5. Let $\left\{h_{l}\right\}_{l \in \mathbb{N}}$ and $\left\{p_{l}\right\}_{l \in \mathbb{N}}$ be given by (1.8). Then for each $l \in \mathbb{N}$,

$$
\left.\partial_{s}^{l} K_{M}(t, s)\right|_{s=0}=h_{l}(t) \text { and }\left.\partial_{s}^{l} K_{M}(t, s)\right|_{s=t}=-p_{l}(t), \quad t \geq 0 .
$$

Proof. Recall the conventional notation: $0^{0}=1$. First of all, we recall that $\mathcal{M}_{j}$ is given in (2.4). Given $t \geq 0$, by $(2.6)$, where $(\alpha, \beta)=(0, l)$, it follows that

$$
\begin{aligned}
\left.\partial_{s}^{l} \mathcal{M}_{j}(t, s)\right|_{s=0} & =\frac{(-1)^{l}}{j !} \sum_{m=0}^{\min \{l, j\}} C_{l}^{m} C_{j}^{m} m !\left(\left.(-s)^{j-m}\right|_{s=0}\right)(\frac{d^{l-m}}{d \tau^{l-m}} \underbrace{M * \cdots * M}_{j})(t) \\
& =\frac{(-1)^{l}}{j !}\left(\chi_{[0, l]}(j) C_{l}^{j} j !\right) \frac{d^{l-j}}{d t^{l-j}} \underbrace{M * \cdots * M}_{j}(t),
\end{aligned}
$$

which, along with (2.3), yields

$$
\left.\partial_{s}^{l} K_{M}(t, s)\right|_{s=0}=\left.\sum_{j=1}^{+\infty} \partial_{s}^{l} \mathcal{M}_{j}(t, s)\right|_{s=0}=(-1)^{l} \sum_{j=0}^{l} C_{l}^{j} \frac{d^{l-j}}{d t^{l-j}} \underbrace{M * \cdots * M}_{j}(t) .
$$

This, along with (1.8), leads to the first equality in (3.13).

Next, from $(2.6)$ with $(\alpha, \beta)=(0, l)$, it follows that

$$
\left.\partial_{s}^{l} \mathcal{M}_{j}(t, s)\right|_{s=t}=\frac{(-1)^{l}}{j !} \sum_{m=0}^{\min \{l, j\}} C_{l}^{m} C_{j}^{m} m !(-t)^{j-m}(\frac{d^{l-m}}{d \tau^{l-m}} \underbrace{M * \cdots * M}_{j})(0) .
$$

Meanwhile, by Lemma 2.1, we find

$$
\frac{d^{l-m}}{d \tau^{l-m}} \underbrace{M * \cdots * M}_{j}(0)=0, \text { when } l-m<j-1 .
$$

From the above and (3.14), we see

$$
\left.\partial_{s}^{l} \mathcal{M}_{j}(t, s)\right|_{s=t}=(-1)^{l} \sum_{m=0}^{\min \{l, j\}} C_{l}^{m} \frac{(-t)^{j-m}}{(j-m) !} \chi_{[0, l-m]}(j-1) \frac{d^{l-m}}{d \tau^{l-m}} \underbrace{M * \cdots * M}_{j}(0) .
$$

This, along with (2.3), yields

$$
\begin{aligned}
\left.\partial_{s}^{l} K_{M}(t, s)\right|_{s=t} & =\left.\sum_{j=1}^{+\infty} \partial_{s}^{l} \mathcal{M}_{j}(t, s)\right|_{s=t} \\
& =(-1)^{l} \sum_{j=1}^{l+1} \sum_{m=0}^{\min \{l, j, l-j+1\}} \frac{(-t)^{j-m}}{(j-m) !}(C_{l}^{l-m} \frac{d^{l-m}}{d \tau^{l-m}} \underbrace{M * \cdots * M}_{j}(0)) .
\end{aligned}
$$

Replacing $j-m$ by a new variable $q$ in the above, using (1.8), we obtain the second equality in (3.13).

Hence, we finish the proof of Lemma 3.5. 
We now are on the position to prove Theorem 3.1 .

Proof of Theorem 3.1. Fix $N \geq 2$ and $\eta>0$ arbitrarily. The proof is structured in three steps. Step 1. Proof of (3.2).

By Lemmas 3.3, 3.4 and 3.5, and by (1.7), we find

$$
\begin{aligned}
w_{\eta}(t)= & e^{-\eta t}+e^{-\eta t} \sum_{l=0}^{N-1}\left(-\left.\partial_{s}^{l} K_{M}(t, s)\right|_{s=t}\right) \eta^{-l-1}+\sum_{l=0}^{N-1}\left(\left.\partial_{s}^{l} K_{M}(t, s)\right|_{s=0}\right) \eta^{-l-1} \\
& +\eta^{-N} \int_{0}^{t} e^{-\eta s} \partial_{s}^{N} K_{M}(t, s) d s \\
= & e^{-\eta t} \sum_{l=0}^{N-1}\left(1+p_{l}(t) \eta^{-l-1}\right)+\sum_{l=0}^{N-1} h_{l}(t) \eta^{-l-1}+R_{N}(t, \eta) \eta^{-N-1}, t \geq 0
\end{aligned}
$$

which leads to $(3.2)$.

Step 2. Proof of conclusion $(i)$.

First, we have

$$
R_{N} \in C\left(\overline{\mathbb{R}^{+}} \times \overline{\mathbb{R}^{+}}\right)
$$

Indeed, we apply Proposition 2.2 to see

$$
K_{M} \in C^{\infty}\left(S_{+}\right)
$$

From (1.7) and (3.16), (3.15) follows at once.

Second, we have

$$
R_{N} \in C\left(\mathbb{R}^{+} ; C\left(\mathbb{R}^{+}\right)\right)
$$

Indeed, it follows from (1.7) that when $t_{2} \geq t_{1}>0$,

$$
\begin{aligned}
& \left\|R_{N}\left(t_{1}, \cdot\right)-R_{N}\left(t_{2}, \cdot\right)\right\|_{C\left(\mathbb{R}^{+}\right)} \\
\leq & \sup _{\tau>0}\left[\int_{0}^{t_{1}} \tau e^{-\tau t}\left|\partial_{s}^{N} K_{M}\left(t_{1}, s\right)-\partial_{s}^{N} K_{M}\left(t_{2}, s\right)\right| d s+\int_{t_{1}}^{t_{2}} \tau e^{-\tau t}\left|\partial_{s}^{N} K_{M}\left(t_{2}, s\right)\right| d s\right] \\
\leq & \left\|\partial_{s}^{N} K_{M}\left(t_{1}, \cdot\right)-\partial_{s}^{N} K_{M}\left(t_{2}, \cdot\right)\right\|_{L^{\infty}\left(0, t_{1}\right)}+\frac{1}{t_{1}} \int_{t_{1}}^{t_{2}}\left|\partial_{s}^{N} K_{M}\left(t_{2}, s\right)\right| d s .
\end{aligned}
$$

This, along with (3.16), yields (3.17).

Third, from (1.7) and Proposition $2.2($ with $(\alpha, \beta)=(0, N))$, we see

$$
\begin{aligned}
\left\|R_{N}(t, \cdot)\right\|_{C\left(\mathbb{R}^{+}\right)} & \leq\left(\sup _{\tau>0} \int_{0}^{t} \tau e^{-\tau s} d s\right)\left\|\partial_{s}^{N} K_{M}(t, \cdot)\right\|_{L^{\infty}(0, t)} \\
& \leq e^{t}\left\{\exp \left[N(1+t)\left(\sum_{j=0}^{N} \max _{0 \leq s \leq t}\left|\frac{d^{j}}{d s^{j}} M(s)\right|\right)\right]-1\right\}, t \geq 0,
\end{aligned}
$$


which leads to (3.3).

Step 3. Proof of conclusion (ii).

Fix any $t \geq 0$. We apply Proposition 2.4 to $\left(t_{0}, s_{0}\right)=(t, 0)$ and $\vec{v}=(0,1) ;\left(t_{0}, s_{0}\right)=(t, t)$ and $\vec{v}=(0,1)$, respectively, to find $j_{1}, j_{2} \in \mathbb{N}$ so that

$$
\partial_{s}^{j_{1}} K_{M}(t, 0) \neq 0 \quad \text { and } \quad \partial_{s}^{j_{2}} K_{M}(t, t) \neq 0
$$

Meanwhile, by (1.8) and (3.13), we have

$$
h_{0} \equiv 0, \partial_{s}^{j_{1}} K_{M}(t, 0)=h_{j_{1}}(t) \text { and } \partial_{s}^{j_{2}} K_{M}(t, t)=-p_{j_{2}}(t) \text {. }
$$

Now, from (3.18) and (3.19), we see that neither $\left\{h_{l}(t)\right\}_{l \geq 1}$ nor $\left\{p_{l}(t)\right\}_{l \in \mathbb{N}}$ is the zero sequence.

This concludes the proof of Theorem 3.1.

\section{Analysis of the memory-flow}

In this section we present several technical propositions describing the nature of each component of the decomposition (1.9). We then prove Theorems 1.1-1.4 one by one. At last we prove some complementary properties of the flow and present an extension of Theorems 1.1-1.2.

\subsection{On the components in the decomposition}

The aim of this subsection is to explain the meaning of each component in the decomposition (1.9), and discuss their regularity properties. This will help us understanding the hybrid parabolic-hyperbolic behavior of the flow more deeply.

The next Proposition 4.1 (which is the conclusion $(i)$ in Theorem 1.2) assures the smoothing effect of the first component in (1.9).

Proposition 4.1. Let $N \geq 2$ be an integer. Then $\mathcal{P}_{N}(t) \mathcal{H}^{-\infty} \subset \mathcal{H}^{+\infty}$ for all $t>0$.

Proof. This directly follows from the definition of $\mathcal{P}_{N}(\cdot)$ (in (1.10)) and the smoothing effect of the heat semigroup $\left\{e^{t A}\right\}_{t \geq 0}$.

The next Proposition 4.2 (which contains the conclusion (ii) in Theorem 1.2) shows the propagation of singularities along the time direction for the second component $\mathcal{W}_{N}(\cdot)$. This shows the hyperbolic nature of $\mathcal{W}_{N}(\cdot)$, with null velocity of propagation.

Proposition 4.2. Let $N \geq 2$ be an integer and let $y_{0} \in \mathcal{H}^{-\infty}$ and $x_{0} \in \Omega$. Then the following statements are equivalent:

(i) For some $t_{0}>0, \mathcal{W}_{N}(\cdot) y_{0} \notin L_{l o c}^{2}\left(t_{0}, x_{0}\right)$;

(ii) For each $t>0, \mathcal{W}_{N}(\cdot) y_{0} \notin L_{l o c}^{2}\left(t, x_{0}\right)$; 
(iii) It holds that $A^{-2} y_{0} \notin L_{l o c}^{2}\left(x_{0}\right)$.

Proof. We first claim that if $z \in \mathcal{H}^{-\infty}$ satisfies $z \in L_{l o c}^{2}\left(x_{0}\right)$, then

$$
A^{-1} z \in L_{l o c}^{2}\left(x_{0}\right)
$$

The proof of (4.1) is classical and, for the sake of completeness, we present it as follows: Write $g:=A^{-1} z$. It is clear that $A g \in L_{l o c}^{2}\left(x_{0}\right)$. Then by (1.3) and some computations, we can see that $\Delta g \in L_{l o c}^{2}\left(x_{0}\right)$. Since $\Delta$ is elliptic at $x_{0}$, one has that $\left.g\right|_{B\left(x_{0}, r\right)} \in H^{2}\left(B\left(x_{0}, r\right)\right)$ for some $r>0$ (see, for instance, [12, Theorem 18.1.29]). Thus, $g \in L_{l o c}^{2}\left(x_{0}\right)$, which gives that $A^{-1} z \in L_{l o c}^{2}\left(x_{0}\right)$.

We next prove that $(i) \Rightarrow(i i i)$. By contradiction, we suppose that $(i)$ is true, but

$$
A^{-2} y_{0} \in L_{l o c}^{2}\left(x_{0}\right)
$$

Since $M$ is analytic, it follows from (1.8) that $h_{0} \equiv 0$ and each $h_{l}$ is smooth. Then by (4.2) and (4.1), one has

$$
\sum_{l=0}^{N-1} h_{l}(\cdot)(-A)^{-l-1} y_{0}=\sum_{l=1}^{N-1} h_{l}(\cdot)(-A)^{-l+1}\left(A^{-2} y_{0}\right) \in L_{l o c}^{2}\left(t, x_{0}\right) \text { for each } t>0 .
$$

This, along with the definition of $\mathcal{W}_{N}(\cdot)$ (in (1.10)), implies that

$$
\mathcal{W}_{N}(\cdot) y_{0} \in L_{l o c}^{2}\left(t, x_{0}\right) \quad \text { for each } \quad t>0
$$

which contradicts $(i)$. Therefore, $(i i i)$ is true.

We now show that $($ iii $) \Rightarrow($ ii $)$. By contradiction, we suppose that $($ iii $)$ holds, but $\mathcal{W}_{N}(\cdot) y_{0} \in$ $L_{l o c}^{2}\left(\hat{t}_{0}, x_{0}\right)$ for some $\hat{t}_{0}>0$. Then from $(1.10)$, we find

$$
\sum_{l=0}^{N-1} h_{l}(\cdot)(-A)^{-l-1} y_{0} \in L_{l o c}^{2}\left(\hat{t}_{0}, x_{0}\right)
$$

We will use (4.3) to prove that

$$
A^{-2} y_{0} \in L_{l o c}^{2}\left(x_{0}\right)
$$

When this is done, we are led to a contradiction with $(\mathrm{iii})$, and then the statement $(\mathrm{ii})$ is true.

To prove (4.4), we observe that there are only two possibilities: either $N=2$ or $N \geq 3$. When that $N=2$, we see from (4.3) and (1.8) that

$$
-M(\cdot) A^{-2} y_{0}=\sum_{l=0}^{N-1} h_{l}(\cdot)(-A)^{-l-1} y_{0} \in L_{l o c}^{2}\left(\hat{t}_{0}, x_{0}\right) .
$$

Since $M$ is nonzero, (4.4) (for this case) follows by integrating the above in the time variable. 
When $N \geq 3$, it follows from (1.8) that $h_{0}=0$ and $h_{1}=-M$. Thus, the leading term of the sum in (4.3) is $-M(\cdot) A^{-2}$. From this and the fact that $M$ is nonzero, by integrating (4.3) in the time variable, we see

$$
A^{-2} y_{0}+\sum_{l=2}^{N-1} c_{l} A^{-l-1} y_{0} \in L_{l o c}^{2}\left(x_{0}\right) \text { for some }\left\{c_{l}\right\}_{l=2}^{N-1} \subset \mathbb{R} .
$$

Write $\mathcal{A}:=-\sum_{l=2}^{N-1} c_{l} A^{-l+1}$. Then the above leads to

$$
f_{0}:=(1-\mathcal{A})\left(A^{-2} y_{0}\right) \in L_{l o c}^{2}\left(x_{0}\right)
$$

Since $y_{0} \in \mathcal{H}^{-\infty}$, there is $m \in \mathbb{N}^{+}$so that $y_{0} \in \mathcal{H}^{-m}$. This, along with (4.5), yields

$$
A^{-2} y_{0}-\left(1+\mathcal{A}+\cdots+\mathcal{A}^{m-1}\right) f_{0}=\mathcal{A}^{m}\left(A^{-2} y_{0}\right) \in L^{2}(\Omega) .
$$

At the same time, since $f_{0} \in L_{l o c}^{2}\left(x_{0}\right)$ in (4.5), it follows from (4.1) that

$$
\mathcal{A}^{j} f_{0} \in L_{l o c}^{2}\left(x_{0}\right) \text { for each } j \in \mathbb{N} \text {. }
$$

Thus, (4.4) (for $N \geq 3$ ) follows from (4.6). Hence, (4.4) is true.

Finally, it is clear that $(i i) \Rightarrow(i)$. Hence, we finish the proof of Proposition 4.2.

The following Proposition 4.3 (which is partially the conclusion (iii) in Theorem 1.2) gives an important time-uniform smoothing effect of the last component $\mathfrak{R}_{N}(\cdot)$.

Proposition 4.3. Let $N \geq 2$ be an integer. Then the last component $\mathfrak{R}_{N}$ in (1.9) exhibits a time-uniform smoothing effect with a gain of $2 N+2$ space derivatives: for each $y_{0} \in \mathcal{H}^{s}$ with $s \in \mathbb{R}$,

$$
\mathfrak{R}_{N}(\cdot) y_{0} \in C\left(\overline{\mathbb{R}^{+}} ; \mathcal{H}^{s+2 N+2}\right) \text {, while } A^{-j} e^{\cdot A} y_{0}, A^{-j} y_{0} \in C\left(\overline{\mathbb{R}^{+}} ; \mathcal{H}^{s+2 j}\right)(0 \leq j \leq N) \text {. }
$$

Remark 4.4. There is a delicate point worth to clarifying. Although the map $t \mapsto A^{-j} e^{t A}$, $t \geq 0$ (for a fixed $j$ ) has infinite order smoothing effect at positive time, it has the finite timeuniform smoothing effect (given in (4.7)) with only $2 j$ space-derivatives gained. This index $2 j$ is optimal due to the following fact: for given $y_{0} \in \mathcal{H}^{-\infty}$ and $s \in \mathbb{R}, A^{-j} e^{\cdot A} y_{0} \in C\left(\overline{\mathbb{R}^{+}} ; \mathcal{H}^{s+2 j}\right)$ if and only if $y_{0} \in \mathcal{H}^{s}$, which can be directly checked.

Proof of Proposition 4.3. The second statement in (4.7) is clearly true. We now show the first statement in (4.7). To this end, we arbitrarily fix $s \in \mathbb{R}, y_{0} \in \mathcal{H}^{s}$ and $t_{0} \geq 0$. We aim to show the continuity of $\mathfrak{R}_{N}(\cdot) y_{0}$ at time $t_{0}$. Fix any $\varepsilon>0$. Write $y_{0}=\sum_{j=1}^{\infty} y_{0, j} e_{j}$. Since $y_{0} \in \mathcal{H}^{s}$, we can choose $j_{\varepsilon} \in \mathbb{N}^{+}$large enough so that $\sum_{j \geq j_{\varepsilon}} \eta_{j}^{s} y_{0, j}^{2}<\varepsilon^{2}$. This, along with the definitions of 
$\mathfrak{R}_{N}(\cdot)$ (see $\left.(1.10)\right)$ and $R_{N}$ (see $(1.7)$ ), yields that for each $t \in\left[0, t_{0}+1\right]$,

$$
\begin{aligned}
& \left\|\Re_{N}(t) y_{0}-\mathfrak{R}_{N}\left(t_{0}\right) y_{0}\right\|_{\mathcal{H}^{s+2 N+2}}^{2} \\
= & \left\|R_{N}(t,-A) y_{0}-R_{N}\left(t_{0},-A\right) y_{0}\right\|_{\mathcal{H}^{s}}^{2} \\
= & \sum_{j \geq 1}\left|R_{N}\left(t, \eta_{j}\right)-R_{N}\left(t_{0}, \eta_{j}\right)\right|^{2} y_{0, j}^{2} \eta_{j}^{s} \\
\leq & \max _{1 \leq j \leq j_{\varepsilon}}\left|R_{N}\left(t, \eta_{j}\right)-R_{N}\left(t_{0}, \eta_{j}\right)\right|^{2}\left(\sum_{j \geq 1} y_{0, j}^{2} \eta_{j}^{s}\right)+4\left\|R_{N}\right\|_{C\left(\left[0, t_{0}+1\right] \times \mathbb{R}^{+}\right)}^{2} \varepsilon^{2}
\end{aligned}
$$

From this and (3.3), one can find some $C>0$ (independent of $\varepsilon$ ) and $\delta \in(0,1)$ so that

$$
\left\|\mathfrak{R}_{N}(t) y_{0}-\mathfrak{R}_{N}\left(t_{0}\right) y_{0}\right\|_{\mathcal{H}^{s+2 N+2}}^{2} \leq C \varepsilon^{2}, \text { when } t \in\left(t_{0}-\delta, t_{0}+\delta\right) \cap \mathbb{R}^{+}
$$

This leads to the continuity of $\mathfrak{R}_{N}(\cdot) y_{0}$ at time $t_{0}$ and ends the proof of Proposition 4.3.

\subsection{Proof of main theorems}

We now give the proofs of Theorems 1.1-1.4 one by one.

Proof of Theorem 1.1. First of all, (1.11) (with $t>0$ ) follows from the conclusion (ii) of Theorem 3.1 at once.

The remainder is to show (1.9). Fix $s \in \mathbb{R}$ and $y_{0} \in \mathcal{H}^{s}$. Recall that $\eta_{j}$ is the $j^{\text {th }}$ eigenvalue of $-A$ and $e_{j}$ is the corresponding normalized eigenfunction in $L^{2}(\Omega)$. Then we can write

$$
y_{0}=\sum_{j \geq 1} y_{0, j} e_{j} \text { and } y\left(t ; y_{0}\right)=\sum_{j \geq 1} y_{j}(t) e_{j}, \quad t \geq 0
$$

where $y_{0, j}:=\left\langle y_{0}, e_{j}\right\rangle_{\mathcal{H}^{s}, \mathcal{H}^{-s}}$ and $y_{j}(\cdot)$ satisfies

$$
y_{j}^{\prime}(t)+\eta_{j} y_{j}(t)+\int_{0}^{t} M(t-\tau) y_{j}(\tau) d \tau=0, \quad t>0 ; \quad y_{j}(0)=y_{0, j} .
$$

By (4.9) and (3.1), we see that for each $j \in \mathbb{N}^{+}$,

$$
y_{j}(t)=y_{0, j} w_{\eta_{j}}(t), \quad t \geq 0,
$$

where $w_{\eta_{j}}(\cdot)$ is the solution to (3.1) with $\eta=\eta_{j}$. Then by (4.8) and (4.10), we have

$$
y\left(t ; y_{0}\right)=\sum_{j \geq 1} w_{\eta_{j}}(t) y_{0, j} e_{j}, \quad t \geq 0
$$

From (4.11) and (3.2) (in Theorem 3.1), we see that when $t \geq 0$,

$$
y\left(t ; y_{0}\right)=\sum_{j \geq 1}\left[e^{-\eta_{j} t}\left(1+\sum_{l=0}^{N-1} p_{l}(t) \eta_{j}^{-l-1}\right)+\sum_{l=0}^{N-1} h_{l}(t) \eta_{j}^{-l-1}+R_{N}\left(t, \eta_{j}\right) \eta_{j}^{-N-1}\right] y_{0, j} e_{j} .
$$


Meanwhile, by functional calculus, we have

$\operatorname{RHS}$ of $(4.12)=\left[\left(e^{t A}+e^{t A} \sum_{l=0}^{N-1} p_{l}(t)(-A)^{-l-1}\right)+\sum_{l=0}^{N-1} h_{l}(t)(-A)^{-l-1}+R_{N}(t,-A)(-A)^{-N-1}\right] y_{0}$.

Here, RHS of (4.12) denotes the expression on the right-hand side of (4.12).

Since $y_{0}$ was arbitrarily taken from $\mathcal{H}^{s}$, we can use (4.12), (4.13), (1.10) and Proposition 7.1 (in Appendix) to get (1.9). This concludes the proof of Theorem 1.1.

Proof of Theorem 1.2. First of all, the conclusions $(i)-(i i)$ in Theorem 1.2 follow from Proposition 4.1 and Proposition 4.2, respectively. In what follows, the conclusions (iii)-(iv) in Theorem 1.2 will be proved one by one.

Step 1. The proof of the conclusion (iii) in Theorem 1.2

By Proposition 4.3, we only need to show (1.13). For this purpose, we first claim

$$
\left.R_{N}(\cdot,-A)\right|_{\mathbb{R}^{+}} \in C\left(\mathbb{R}^{+} ; \mathcal{L}\left(\mathcal{H}^{s}\right)\right) \text { for each } s \in \mathbb{R} \text {. }
$$

To prove (4.14), we arbitrarily fix $s \in \mathbb{R}$ and $t_{2} \geq t_{1}>0$. Set

$$
\widetilde{\mathcal{R}}(-A):=R_{N}\left(t_{2},-A\right)-R_{N}\left(t_{1},-A\right) .
$$

Then $\widetilde{\mathcal{R}}(-A)$ is the operator obtained by the functional calculus of the function: $R_{N}\left(t_{2}, \cdot\right)-$ $R_{N}\left(t_{1}, \cdot\right)$. By $(1.4)$ and by the spectral representation of $\widetilde{\mathcal{R}}(-A)$, we see

$$
\|\widetilde{\mathcal{R}}(-A)\|_{\mathcal{L}\left(\mathcal{H}^{s}\right)}^{2}=\sup _{\sum \eta_{j}^{s} z_{j}^{2} \leq 1}\left\|\sum_{j \geq 1} \widetilde{\mathcal{R}}\left(\eta_{j}\right) z_{j} e_{j}\right\|_{\mathcal{H}^{s}}^{2}=\sup _{\sum \eta_{j}^{s} z_{j}^{2} \leq 1} \sum_{j=1}^{+\infty} \eta_{j}^{s}\left|\widetilde{\mathcal{R}}\left(\eta_{j}\right)\right|^{2} z_{j}^{2} \leq \sup _{\eta>0}|\widetilde{\mathcal{R}}(\eta)|^{2} .
$$

This, along with (4.15), yields

$$
\left\|R_{N}\left(t_{2},-A\right)-R_{N}\left(t_{1},-A\right)\right\|_{\mathcal{L}\left(\mathcal{H}^{s}\right)}=\|\widetilde{\mathcal{R}}(-A)\|_{\mathcal{L}\left(\mathcal{H}^{s}\right)} \leq\left\|R_{N}\left(t_{2}, \cdot\right)-R_{N}\left(t_{1}, \cdot\right)\right\|_{C\left(\mathbb{R}^{+}\right)} .
$$

Since $R_{N} \in C\left(\mathbb{R}^{+} ; C\left(\mathbb{R}^{+}\right)\right.$) (see Theorem 3.1), (4.14) follows from (4.16) at once.

We now show (1.13). Indeed, arguing as in the proof of (4.16), we can obtain

$$
\left\|R_{N}(t,-A)\right\|_{\mathcal{L}\left(\mathcal{H}^{s}\right)} \leq\left\|R_{N}(t, \cdot)\right\|_{C\left(\mathbb{R}^{+}\right)}, \quad t \geq 0
$$

This, along with (3.3) (in Theorem 3.1), leads to (1.13).

Hence, the conclusion (

Step 2. The proof of the conclusion (iv) in Theorem 1.2

Arbitrarily fix $y_{0} \in \mathcal{H}^{-\infty}, x_{0} \in \Omega$ and $t>0$. There is an integer $m \geq 2$ so that

$$
y_{0} \in \mathcal{H}^{-2 m} \text {. }
$$


Then by (1.9) and (1.13) (where $N$ is replaced by $m$ ), one can easily check

$$
\Phi(\cdot) y_{0}-\mathcal{P}_{m}(\cdot) y_{0}-\mathcal{W}_{m}(\cdot) y_{0} \in L_{l o c}^{\infty}\left(\overline{\mathbb{R}^{+}} ; L^{2}(\Omega)\right) .
$$

Combine (4.17) and Proposition 4.1 (where $N$ is replaced by $m$ ) to get

$$
\Phi(\cdot) y_{0} \notin L_{l o c}^{2}\left(t, x_{0}\right) \Leftrightarrow \mathcal{W}_{m}(\cdot) y_{0} \notin L_{l o c}^{2}\left(t, x_{0}\right) .
$$

Meanwhile, for each integer $k \geq 2$, it follows by Proposition 4.2 (where $N$ is replaced by $k$ ) that

$$
\mathcal{W}_{k}(\cdot) y_{0} \notin L_{l o c}^{2}\left(t, x_{0}\right) \Leftrightarrow A^{-2} y_{0} \notin L_{l o c}^{2}\left(x_{0}\right),
$$

in particular,

$$
\mathcal{W}_{m}(\cdot) y_{0} \notin L_{l o c}^{2}\left(t, x_{0}\right) \Leftrightarrow \mathcal{W}_{N}(\cdot) y_{0} \notin L_{l o c}^{2}\left(t, x_{0}\right)
$$

This, along with (4.18), yields

$$
\Phi(\cdot) y_{0} \notin L_{l o c}^{2}\left(t, x_{0}\right) \Leftrightarrow \mathcal{W}_{N}(\cdot) y_{0} \notin L_{l o c}^{2}\left(t, x_{0}\right) .
$$

Thus, the conclusion (iv) follows from (4.19) and Proposition 4.2 at once.

Hence, we complete the proof of Theorem 1.2.

Proof of Theorem 1.3. Arbitrarily fix an integer $N \geq 2$. We first show (1.15). Indeed, one can see from (1.2), (1.10) and (1.7) that for each $j \in \mathbb{N}^{+}$,

$$
\begin{aligned}
& \Phi(0) e_{j}=e_{j}, \mathcal{P}_{N}(0) e_{j}=\left(1+\sum_{l=0}^{N-1} p_{l}(0) \eta_{j}^{-l-1}\right) e_{j}, \\
& \mathfrak{R}_{N}(0) e_{j}=0, \mathcal{W}_{N}(0) e_{j}=\left(\sum_{l=0}^{N-1} h_{l}(0) \eta_{j}^{-l-1}\right) e_{j} .
\end{aligned}
$$

Since $\lim _{j \rightarrow+\infty} \eta_{j}=+\infty$, the above leads to $(1.15)$ at once.

We next prove (1.16). Arbitrarily fix $t>0$. On one hand, it follows from (1.8) that $h_{0} \equiv 0$ and $h_{1}(t)=-M(t)$, which, along with (1.10), yield

$$
\mathcal{W}_{N}(t) e_{j}=-M(t) \eta_{j}^{-2} e_{j}+\sum_{1<l \leq N-1} h_{l}(t) \eta_{j}^{-l-1} e_{j} \text { for all } j \geq 1
$$

This implies

$$
\lim _{j \rightarrow+\infty}\left\|\mathcal{W}_{N}(t) e_{j}\right\|_{\mathcal{H}^{4}}=|M(t)| \text { and } \lim _{j \rightarrow+\infty}\left\|\mathcal{W}_{N}(t) e_{j}\right\|_{\mathcal{H}^{s}}=0, \forall s<4
$$

On the other hand, from (1.10), it follows that for each $j \geq 1$,

$$
\mathcal{P}_{N}(t) e_{j}=e^{-t \eta_{j}}\left(1+\sum_{l=0}^{N-1} p_{l}(t) \eta_{j}^{-l-1}\right) e_{j} \text { and } \mathfrak{R}_{N}(t) e_{j}=\eta_{j}^{-N-1} R_{N}(t,-A) e_{j} .
$$


Since $\lim _{j \rightarrow+\infty} \eta_{j}^{-1}=\lim _{j \rightarrow+\infty} \eta_{j}^{\frac{s}{2}} e^{-t \eta_{j}}=0(s \in \mathbb{R})$, the above, together with (1.13), gives

$$
\lim _{j \rightarrow+\infty}\left\|\mathcal{P}_{N}(t) e_{j}\right\|_{\mathcal{H}^{s_{1}}}=\lim _{j \rightarrow+\infty}\left\|\Re_{N}(t) e_{j}\right\|_{\mathcal{H}^{s_{2}}}=0, s_{1} \in \mathbb{R}, s_{2}<2 N+2 .
$$

Now, by (4.20) and (4.21), we can use the decomposition (1.9) (with the above $N \geq 2$ ) to get

$$
\lim _{j \rightarrow+\infty}\left\|\Phi(t) e_{j}\right\|_{\mathcal{H}^{4}}=|M(t)| \text { and } \lim _{j \rightarrow+\infty}\left\|\Phi(t) e_{j}\right\|_{\mathcal{H}^{s}}=0, \forall s<4 .
$$

These, along with (4.20)-(4.21), lead to (1.16). This completes the proof of Theorem 1.3.

Proof of Theorem 1.4. Arbitrarily fix $s \in \mathbb{R}$. We will prove the conclusions $(i)$-(iii) one by one.

(i) Arbitrarily fix $\alpha \in[0,4]$ and $t>0$. We apply (1.9) (with $N=2$ ), as well as (1.10) and (1.8), to obtain

$$
\Phi(t)=\left(e^{t A}-p_{0}(t) A^{-1} e^{t A}+p_{1}(t) A^{-2} e^{t A}\right)-M(t) A^{-2}+R_{2}(t,-A)(-A)^{-3} .
$$

Meanwhile, notice that

$$
\left\|A^{\frac{\alpha}{2}-j} e^{t A}\right\|_{\mathcal{L}\left(\mathcal{H}^{s}\right)}\left(=\sup _{j \in \mathbb{N}^{+}} \eta_{j}^{\frac{\alpha}{2}-j} e^{-t \eta_{j}}\right) \leq t^{j-\frac{\alpha}{2}} \text { and }\left\|A^{\frac{\alpha}{2}-j-2}\right\|_{\mathcal{L}\left(\mathcal{H}^{s}\right)} \leq \eta_{1}^{\frac{\alpha}{2}-j-2}, j=0,1,2
$$

These, along with (4.22), yield

$$
\|\Phi(t)\|_{\mathcal{L}\left(\mathcal{H}^{s}, \mathcal{H}^{s+\alpha}\right)} \leq t^{-\frac{\alpha}{2}}\left(1+t\left|p_{0}(t)\right|+t^{2}\left|p_{1}(t)\right|\right)+\eta_{1}^{\frac{\alpha}{2}-2}|M(t)|+\eta_{1}^{\frac{\alpha}{2}-3}\left\|R_{2}(t, \cdot)\right\|_{\mathcal{L}\left(\mathcal{H}^{s+\alpha}\right)} .
$$

After direct computations, we obtain (1.17) from the above, (1.8) and (1.13) (where $N=2$ ).

(ii) Assume that (1.18) holds for $t \in\left(0, \delta_{0}\right)$. We first claim

$$
+\infty>\limsup _{j \rightarrow+\infty} \eta_{j}^{\frac{\alpha_{0}}{2}-2} \text {, i.e., } \alpha_{0} \leq 4 .
$$

Indeed, by the assumption $(\mathfrak{C})$, we can choose small $t_{0} \in\left(0, \delta_{0}\right)$ so that

$$
M\left(t_{0}\right) \neq 0
$$

Then from (4.22) and (1.13) (where $N=2$ ), it follows that for each $j \geq 1$,

$$
\left\|\Phi\left(t_{0}\right)\right\|_{\mathcal{L}\left(\mathcal{H}^{s}, \mathcal{H}^{s+\alpha_{0}}\right)} \geq\left\|\Phi\left(t_{0}\right) e_{j}\right\|_{\mathcal{H}^{s+\alpha_{0}}} /\left\|e_{j}\right\|_{\mathcal{H}^{s}}=\eta_{j}^{\frac{\alpha_{0}}{2}-2}\left(\left|M\left(t_{0}\right)\right|+O\left(\eta_{j}^{-1}\right)\right),
$$

(Here and what follows, by $O\left(\eta_{j}^{-1}\right)$ with $j \geq 1$, we mean that there is $C_{1}>0$, independent of $j$, so that $\left|O\left(\eta_{j}^{-1}\right)\right| \leq C_{1} \eta_{j}^{-1}$.) This, along with (1.18) (where $t=t_{0}$ ), (4.24) and the fact that $\lim _{j \rightarrow+\infty} \eta_{j}=+\infty$, leads to $(4.23)$.

We next claim

$$
\liminf _{t \rightarrow 0^{+}} t^{\frac{\alpha_{0}}{2}}\|\Phi(t)\|_{\mathcal{L}\left(\mathcal{H}^{s}, \mathcal{H}^{\left.s+\alpha_{0}\right)}\right.}>0
$$


In fact, from (1.18) (where $\left.z=e_{j}\right),(4.22)$ and (1.13), we see that when $t \in\left(0, \delta_{0}\right)$ and $j \in \mathbb{N}^{+}$,

$$
\begin{aligned}
t^{\frac{\alpha_{0}}{2}}\|\Phi(t)\|_{\mathcal{L}\left(\mathcal{H}^{s}, \mathcal{H}^{\left.s+\alpha_{0}\right)}\right.} & \geq t^{\frac{\alpha_{0}}{2}}\left\|\Phi(t) e_{j}\right\|_{\mathcal{H}^{s+\alpha_{0}}} /\left\|e_{j}\right\|_{\mathcal{H}^{s}} \\
& \geq\left(t \eta_{j}\right)^{\frac{\alpha_{0}}{2}}\left(\left|1+O\left(\eta_{j}^{-1}\right)\right| e^{-t \eta_{j}}-O\left(\eta_{j}^{-1}\right)\right) .
\end{aligned}
$$

Meanwhile, it follows by Weyl's asymptotic formula for the eigenvalues (see for instance [16, XIII.15]) that $\lim _{j \rightarrow+\infty} \eta_{j+1} / \eta_{j}=1$. Thus, there is $j_{0} \in \mathbb{N}^{+}$so that

$$
\left[\eta_{j_{0}},+\infty\right)=\cup_{j \geq j_{0}}\left[\eta_{j}, 2 \eta_{j}\right)
$$

Therefore, for each $t \in\left(0, \eta_{j_{0}}^{-1}\right]$, there is an integer $j_{t} \geq j_{0}$ so that

$$
t^{-1} \in\left[\eta_{j_{t}}, 2 \eta_{j_{t}}\right) \text {, i.e., } \frac{1}{2}<t \eta_{j_{t}} \leq 1 .
$$

This, along with (4.26), leads to (4.25).

Finally, the conclusion (ii) follows from (4.23) and (4.25) at once.

(iii) We first claim

$$
\Phi(\cdot) y_{0} \in C\left(\mathbb{R}^{+} ; \mathcal{H}^{s+4}\right) \text { for each } y_{0} \in \mathcal{H}^{s}
$$

To this end, we arbitrarily fix $y_{0} \in \mathcal{H}^{s}, t_{0}>0$ and $\varepsilon>0$. Since $y_{0} \in \mathcal{H}^{s}$, there is $j_{\varepsilon} \in \mathbb{N}^{+}$so that

$$
\left\|y_{0}-y_{0, j_{\varepsilon}}\right\|_{\mathcal{H}^{s}}<\varepsilon \text { where } y_{0, j_{\varepsilon}}:=\sum_{j=1}^{j_{\varepsilon}}\left\langle y_{0}, e_{j}\right\rangle_{\mathcal{H}^{s}, \mathcal{H}^{-s}} e_{j}
$$

At the same time, one can easily check $\Phi(\cdot) y_{0, j_{\varepsilon}} \in C\left(\mathbb{R}^{+} ; \mathcal{H}^{s+4}\right)$, which implies there is $\delta_{\varepsilon} \in$ $\left(0, t_{0} / 2\right)$ so that

$$
\sup _{t_{0}-\delta_{\varepsilon}<t<t_{0}+\delta_{\varepsilon}}\left\|\Phi(t) y_{0, j_{\varepsilon}}-\Phi\left(t_{0}\right) y_{0, j_{\varepsilon}}\right\|_{\mathcal{H}^{s+4}}<\varepsilon .
$$

This, together with (4.28), gives

$$
\sup _{t_{0}-\delta_{\varepsilon}<t<t_{0}+\delta_{\varepsilon}}\left\|\Phi(t) y_{0}-\Phi\left(t_{0}\right) y_{0}\right\|_{\mathcal{H}^{s+4}}<\varepsilon+2 \sup _{t_{0} / 2 \leq \tau \leq 2 t_{0}}\|\Phi(\tau)\|_{\mathcal{L}\left(\mathcal{H}^{s}, \mathcal{H}^{s+4}\right)} \varepsilon
$$

which, along with (1.17), leads to (4.27).

We next claim that for each $\alpha>4$,

$$
\Phi(\cdot) \hat{y}_{0} \notin C\left(\mathbb{R}^{+} ; \mathcal{H}^{s+\alpha}\right) \text { for some } \hat{y}_{0} \in \mathcal{H}^{s}
$$

For this purpose, we arbitrarily fix $\alpha>4$. Since $h_{0}(t) \equiv 0$ and $h_{1}(t)=-M(t)$ (see (1.8)), we apply (1.9) (with $N=2$ ), as well as (1.10) and (1.13), to obtain

$$
\lim _{j \rightarrow+\infty} \eta_{j}^{2}\left\langle\Phi(t) e_{j}, e_{j}\right\rangle_{L^{2}(\Omega)}=-M(t) \text { for each } t>0
$$


Meanwhile, by the assumption $(\mathfrak{C})$, we can choose $\hat{t}_{0}>0$ so that $M\left(\hat{t}_{0}\right) \neq 0$. This, along with (4.30) and the fact that $\alpha>4$, yields

$$
\eta_{j}^{\frac{\alpha}{2}}\left|\left\langle\Phi\left(\hat{t}_{0}\right) e_{j}, e_{j}\right\rangle_{L^{2}(\Omega)}\right| \rightarrow+\infty \text { as } j \rightarrow+\infty
$$

Thus we can choose a subsequence $\left\{k_{j}\right\}_{j \geq 1}$ of $\mathbb{N}^{+}$so that

$$
\eta_{k_{j}}^{\frac{\alpha}{2}}\left|\left\langle\Phi\left(\hat{t}_{0}\right) e_{k_{j}}, e_{k_{j}}\right\rangle_{L^{2}(\Omega)}\right| \geq j 2^{j} \text { for each } j \geq 1
$$

We now define $\hat{y}_{0} \in \mathcal{H}^{s}$ as follows:

$$
\hat{y}_{0}:=\sum_{j \geq 1} 2^{-j} \eta_{k_{j}}^{-\frac{s}{2}} e_{k_{j}} .
$$

Then, by (4.31) and (4.32), we find

$$
\begin{aligned}
\left\|\Phi\left(\hat{t}_{0}\right) \hat{y}_{0}\right\|_{\mathcal{H}^{s+\alpha}}^{2} & =\sum_{j \geq 1} \eta_{k_{j}}^{s+\alpha}\left(\left\langle\Phi\left(\hat{t}_{0}\right) e_{k_{j}}, e_{k_{j}}\right\rangle_{L^{2}(\Omega)}\left\langle\hat{y}_{0}, e_{k_{j}}\right\rangle_{\mathcal{H}^{s}, \mathcal{H}^{-s}}\right)^{2} \\
& =\sum_{j \geq 1}\left(\eta_{k_{j}}^{\frac{\alpha}{2}}\left\langle\Phi\left(\hat{t}_{0}\right) e_{k_{j}}, e_{k_{j}}\right\rangle_{L^{2}(\Omega)} 2^{-j}\right)^{2} \geq \sum_{j \geq 1} j^{2}=+\infty
\end{aligned}
$$

which implies $\Phi\left(\hat{t}_{0}\right) \hat{y}_{0} \notin \mathcal{H}^{s+\alpha}$. This leads to (4.29).

In conclusion, we complete the proof of Theorem 1.4.

Remark 4.5. By a very similar way to that used in the proof of (1.17) (in Theorem 1.4), we can also show what follows: For each $t>0$,

$$
\Phi(t) \in \mathcal{L}\left(\mathcal{H}^{s}, \mathcal{H}^{s+2 k(t)+2}\right) \text { for each } s \in \mathbb{R}
$$

where $k(t):=\min \left\{l \geq 1: h_{l}(t) \neq 0\right\}$.

From (4.33) and the fact that $h_{1}(t)=-M(t)$ (which follows from (1.8)), we conclude that the smoothing effect of the flow $\Phi(t)$ at points in the set $\{t>0: M(t)=0\}$ is better than that at points in the set $\{t>0: M(t) \neq 0\}$.

\subsection{Other properties of the flow and the components}

This subsection presents more properties of the flow and the components. More precisely, first, we formulate Propositions 4.6 to illustrate how the component $\mathcal{P}_{N}$ influences the flow, from the perspective of the singularities; (In plain language, it tells us that the singularity of $\mathcal{P}_{N}(0)$ determines the singularity of $\Phi(0)$.); second, we give Proposition 4.7 to show that both $\mathcal{P}_{N}(0)$ and $\mathcal{W}_{N}(0)$ are not projections; last, we present Propositions 4.8-4.9, which might have independent interest.

Proposition 4.6. Let $N \geq 2$ be an integer and let $y_{0} \in \mathcal{H}^{-\infty}$ and $x_{0} \in \Omega$. Then $\left(y_{0}=\right) \Phi(0) y_{0} \notin$ $L_{l o c}^{2}\left(x_{0}\right)$ if and only if $\mathcal{P}_{N}(0) y_{0} \notin L_{l o c}^{2}\left(x_{0}\right)$. 
Proof. First of all, since $\Phi(0) y_{0}=y_{0}$, we have

$$
\Phi(0) y_{0} \notin L_{l o c}^{2}\left(x_{0}\right) \Leftrightarrow y_{0} \notin L_{l o c}^{2}\left(x_{0}\right) .
$$

We claim

$$
\mathcal{P}_{N}(0) y_{0} \notin L_{l o c}^{2}\left(x_{0}\right) \Leftrightarrow y_{0} \notin L_{l o c}^{2}\left(x_{0}\right)
$$

When this is proved, the conclusion in this proposition follows from (4.34) and (4.35) at once.

We now show (4.35). To prove the necessity, we suppose, by contradiction, that the statement on the left-hand side of $(4.35)$ is true, but $y_{0} \in L_{l o c}^{2}\left(x_{0}\right)$. Then we apply (4.1) to obtain that

$$
A^{-j} y_{0} \in L_{l o c}^{2}\left(x_{0}\right) \text { for each } j \in \mathbb{N} \text {. }
$$

At the same time, it follows from (1.10) that

$$
\mathcal{P}_{N}(0) y_{0}=y_{0}+\sum_{l=0}^{N-1} p_{l}(0)(-A)^{-l-1} y_{0} .
$$

These imply that $\mathcal{P}_{N}(0) y_{0} \in L_{l o c}^{2}\left(x_{0}\right)$, which contradicts the statement on the left-hand side of (4.35). Therefore, we have shown the necessity.

To show the sufficiency, we suppose, by contradiction, that the statement on the right-hand side of (4.35) holds, but $\mathcal{P}_{N}(0) y_{0} \in L_{\text {loc }}^{2}\left(x_{0}\right)$. Then from $(1.10)$, there is a sequence $\left(\hat{c}_{l}\right)_{l=0}^{N-1} \subset \mathbb{R}$ so that

$$
y_{0}+\sum_{l=0}^{N-1} \hat{c}_{l} A^{-l-1} y_{0} \in L_{l o c}^{2}\left(x_{0}\right) .
$$

Then by the similar way as that used in the proof of "(4.3) $\Rightarrow(4.4)$ ", one can get that $y_{0} \in L_{l o c}^{2}\left(x_{0}\right)$. This contradicts the statement on the right-hand side of (4.35). Therefore, the sufficiency is proved. Hence, we finish the proof of Proposition 4.6.

Proposition 4.7. The following conclusions are true:

(i) For each integer $N \geq 2$,

$$
\mathfrak{R}_{N}(0)=0 \text { and } y_{0}=\mathcal{P}_{N}(0) y_{0}+\mathcal{W}_{N}(0) y_{0} \text { for each } y_{0} \in L^{2}(\Omega)
$$

(ii) For each integer $N \geq 2, \mathcal{P}_{N}(0)$ and $\mathcal{W}_{N}(0)$ are projections over $L^{2}(\Omega)$ if and only if

$$
M(0)=\cdots=M^{(j)}(0)=\cdots=M^{(N-2)}(0)=0 .
$$

Proof. First, the conclusion $(i)$ follows from (1.10), (1.7) and (1.9) (with $t=0$ ) at once.

We next prove the conclusion $(i i)$. To show the sufficiency, we assume that (4.37) is true. Then by (4.37), we can apply (2.1) (with $t \rightarrow 0^{+}$) to get

$$
\frac{d^{l}}{d t^{l}} \underbrace{M * \cdots * M}_{j}(0)=0, j \in \mathbb{N}^{+}, l \in\{0, \ldots, N-2\} .
$$


This, along with (1.8), yields

$$
h_{l}(0)=0, \quad 0 \leq l \leq N-1
$$

from which and (1.10), it follows $\mathcal{W}_{N}(0)=0$ and consequently, $\mathcal{W}_{N}(0)$ is a projection. Then by the second equality in (4.36), we see that $\mathcal{P}_{N}(0)$ is a projection.

To prove the necessity, we suppose that $\mathcal{P}_{N}(0)$ and $\mathcal{W}_{N}(0)$ are projections over $L^{2}(\Omega)$. Then we have

$$
\mathcal{W}_{N}(0)^{2} y_{0}=\mathcal{W}_{N}(0) y_{0} \text { for each } y_{0} \in L^{2}(\Omega)
$$

Taking $y_{0}=e_{j}$ with $j \in \mathbb{N}^{+}$in the above and using (1.10), we find

$$
\begin{aligned}
\left(\sum_{l=0}^{N-1} h_{l}(0) \eta_{j}^{-l-1}\right)^{2} e_{j} & =\left(\sum_{l=0}^{N-1} h_{l}(0)(-A)^{-l-1}\right)^{2} e_{j}=\mathcal{W}_{N}(0)^{2} y_{0} \\
& =\mathcal{W}_{N}(0) y_{0}=\left(\sum_{l=0}^{N-1} h_{l}(0)(-A)^{-l-1}\right) e_{j}=\left(\sum_{l=0}^{N-1} h_{l}(0) \eta_{j}^{-l-1}\right) e_{j} .
\end{aligned}
$$

This implies

$$
\left(\sum_{l=0}^{N-1} h_{l}(0) \eta_{j}^{-l-1}\right)^{2}=\sum_{l=0}^{N-1} h_{l}(0) \eta_{j}^{-l-1} \text { for all } j \geq 1 \text {. }
$$

Since $\lim _{j \rightarrow+\infty} \eta_{j}=+\infty$, the above, divided by $\eta_{j}^{-1}, \ldots, \eta_{j}^{-N}$ respectively, gives $h_{l}(0)=0,0 \leq$ $l \leq N-1$. Then by direct computations and by (1.8), we get (4.37). This ends the proof of Proposition 4.7.

Proposition 4.8. Let $K_{M}$ be given by (1.6). Then

$$
\Phi(t)^{*}=\Phi(t)=e^{t A}+\int_{0}^{t} K_{M}(t, \tau) e^{\tau A} d \tau, \quad t \geq 0
$$

Moreover, it holds that

$$
\sup _{s \in \mathbb{R}}\left\|\Phi(t)-e^{t A}\right\|_{\mathcal{L}\left(\mathcal{H}^{s}\right)} \leq \inf _{\lambda \geq-\eta_{1}} e^{\lambda t}\left[\exp \left(t \int_{0}^{t} e^{-\lambda \tau}|M(\tau)| d \tau\right)-1\right], \quad t \geq 0
$$

(Here, $-\eta_{1}$ is the first eigenvalue of $A$.)

Proof. Arbitrarily fix $s \in \mathbb{R}$ and $y_{0} \in \mathcal{H}^{s}$. By (4.8), (4.11) and Lemma 3.3, we find

$$
y\left(t ; y_{0}\right)=\sum_{j \geq 1} w_{\eta_{j}}(t) y_{0, j} e_{j}=\sum_{j \geq 1}\left(e^{-\eta_{j} t}+\int_{0}^{t} K_{M}(t, \tau) e^{-\eta_{j} \tau} d \tau\right) y_{0, j} e_{j}, \quad t \geq 0 .
$$

Now the second equality in (4.38) follows from (4.40) and (1.2), while the first equality in (4.38) follows by the second one in (4.38) and the fact that for each $t \geq 0, e^{t A}=e^{t A^{*}}$ in $\mathcal{L}\left(\mathcal{H}^{s}\right)$. 
Finally, it follows from (4.38) that

$$
\left\|\Phi(t)-e^{t A}\right\|_{\mathcal{L}\left(\mathcal{H}^{s}\right)} \leq \int_{0}^{t}\left\|e^{\tau A}\right\|_{\mathcal{L}\left(\mathcal{H}^{s}\right)}\left|K_{M}(t, \tau)\right| d \tau \leq \int_{0}^{t} e^{-\eta_{1} \tau}\left|K_{M}(t, \tau)\right| d \tau, t \geq 0 .
$$

This, along with (2.14) in Proposition 2.6, where $\lambda \geq-\eta_{1}$, yields

$$
\left\|\Phi(t)-e^{t A}\right\|_{\mathcal{L}\left(\mathcal{H}^{s}\right)} \leq e^{\lambda t} \int_{0}^{t} e^{-\lambda(t-\tau)}\left|K_{M}(t, \tau)\right| d \tau \leq e^{\lambda t}\left[\exp \left(t \int_{0}^{t} e^{-\lambda \tau}|M(\tau)| d \tau\right)-1\right], t \geq 0,
$$

which leads to (4.39) and completes the proof of Proposition 4.8.

Proposition 4.9. Let $s \in \mathbb{R}$. Then $\Phi(\cdot)$ is real analytic from $\mathbb{R}^{+}$to $\mathcal{L}\left(\mathcal{H}^{s}\right)$.

Proof. According to Proposition 2.3, $K_{M}$ is real analytic over $S_{+}$. Then by (4.38) and the analyticity of $\left\{e^{t A}\right\}_{t>0}$, we obtain that $\Phi(\cdot)$ is real analytic from $\mathbb{R}^{+}$to $\mathcal{L}\left(\mathcal{H}^{s}\right)$. This ends the proof of Proposition 4.9.

\subsection{Less regular memory kernels}

The techniques of this paper can also be employed to handle less regular memory kernels. Assume that:

$\left(\mathfrak{C}_{1}\right) \quad$ The memory kernel $M$ is in $C^{N_{0}}\left(\overline{\mathbb{R}^{+}}\right)$for a fixed integer $N_{0} \geq 2$.

Then, the following holds, and can be proved by the same arguments of the proofs of Theorems $1.1-1.2$.

Theorem 4.10. Suppose that $\left(\mathfrak{C}_{1}\right)$ is true. Then for each $N \in\left\{2, \ldots, N_{0}\right\}$, all results of Theorems 1.1-1.2, except for (1.11)-(1.12) and (1.14), are true.

\section{An example}

In this section we analyze with more details the decomposition (1.9) in the particular case of the following memory kernel:

$$
M(t)=\alpha e^{\lambda t}, \quad t \geq 0, \text { where } \lambda, \alpha \in \mathbb{R}, \text { with } \alpha \neq 0 .
$$

In what follows, we adopt the conventional notation: $0^{0}:=1$ and $\sum_{\emptyset} \cdot:=0$.

In this particular case, we have the following explicit expressions: For each $l \in \mathbb{N}$,

$$
\left\{\begin{array}{l}
h_{l}(t)=e^{\lambda t}(-1)^{l} \sum_{\substack{m, k \in \mathbb{N}, j \in \mathbb{N}^{+} \\
k-m+2 j=l+1 \\
m+k \leq l-1}} C_{l}^{l-j} \alpha^{j} \lambda^{k} \frac{t^{m}}{m !}, t \geq 0, \\
p_{l}(t)=(-1)^{l+1} \sum_{\substack{m, k \in \mathbb{N}, j \in \mathbb{N}^{+} \\
k-m+2 j=l+1, m+k \leq l+1}} C_{l}^{l-j+m} \alpha^{j} \lambda^{k} \frac{(-t)^{m}}{m !}, t \geq 0, \\
R_{N}(t, \tau)=(-1)^{N} N ! \int_{0}^{t} \tau e^{-\tau s} e^{\lambda(t-s)} \mathcal{F}_{N}(t, s) d s, \quad t, \tau \geq 0,
\end{array}\right.
$$


where

$$
\mathcal{F}_{N}(t, s):=\sum_{\substack{\beta_{1}, \beta_{2}, \beta_{3} \in \mathbb{N}, j \in \mathbb{N}^{+} \\ \beta_{1}+\beta_{2}+\beta_{3}=N, \beta_{1} \leq j, \beta_{2} \leq j-1}} \frac{(-s)^{j-\beta_{1}}}{\left(j-\beta_{1}\right) !} \frac{(t-s)^{j-1-\beta_{2}}}{\left(j-1-\beta_{2}\right) !} \lambda^{\beta_{3}} \alpha^{j} .
$$

For this example, the following holds:

Proposition 5.1. For almost every $t \geq 0$ and for each $l \in \mathbb{N}^{+}$, it holds that

$$
h_{l}(t) \neq 0 \text { and } p_{l}(t) \neq 0 \text {. }
$$

Proof. Fix $l \geq 1$ arbitrarily. It follows from (5.1) that the function $t \mapsto e^{-\lambda t} h_{l}(t)(t \geq 0)$ is a polynomial of the order $l-1$ and satisfies

$$
e^{-\lambda t} h_{l}(t)=(-\alpha)^{l} \frac{t^{l-1}}{(l-1) !}+\sum_{m=0}^{l-2} \cdots t^{m}, \quad t \geq 0 .
$$

Thus $h_{l}(t) \neq 0$ for a.e. $t \geq 0$, which implies the first inequality in (5.2). The same can be done for $p_{l}$ by the similar argument. This ends the proof.

Remark 5.2. The following comments on Proposition 5.1 are worth considering.

(R1) As $N$ increases, for almost every $t \geq 0$, both $\mathcal{P}_{N}(t)$ and $\mathcal{W}_{N}(t)$ involve more and more nontrivial terms.

(R2) For each $N \in \mathbb{N}^{+}$and for a.e. $t \geq 0$, the remainder $\mathfrak{R}_{N}(t)$ inherits a hybrid heat/wave structure (and thus is not negligible). Indeed, from (1.9)-(1.10), one can directly obtain the following recursive equality:

$$
\mathfrak{R}_{j}(t)=\mathfrak{R}_{j+1}(t)+p_{j}(t) e^{t A}(-A)^{-j-1}+h_{j}(t)(-A)^{-j-1}, t \geq 0, j \in \mathbb{N}^{+} .
$$

From this and (5.2), it follows that for each $N \in \mathbb{N}^{+}$and for a.e. $t \geq 0, \Re_{N}(t)$ contains both nontrivial terms in $\mathcal{P}_{N+1}(t)$ and $\mathcal{W}_{N+1}(t)$, and thus inherits the hybrid structure.

(R3) Initial data of the form $\mathcal{P}_{N}(0) y_{0}\left(\operatorname{resp} ., \mathcal{W}_{N}(0) y_{0}\right)$, under the action of the flow $\Phi(\cdot)$, may lead to three nontrivial components. More precisely, given a fixed integer $N \geq 2$, there is $j \in \mathbb{N}^{+}$so that the following equality

$$
\begin{aligned}
\Phi(t)\left(\mathcal{P}_{N}(0) e_{j}\right) & =I_{N, 1}(t)+I_{N, 2}(t)+I_{N, 3}(t) \\
& :=\mathcal{P}_{N}(t)\left(\mathcal{P}_{N}(0) e_{j}\right)+\mathcal{W}_{N}(t)\left(\mathcal{P}_{N}(0) e_{j}\right)+\Re_{N}(t)\left(\mathcal{P}_{N}(0) e_{j}\right), \quad t \geq 0
\end{aligned}
$$

has the property

$$
I_{N, 1}(t), I_{N, 2}(t), I_{N, 3}(t) \neq 0 \text { in } L^{2}(\Omega) \text { for a.e. } t \geq 0 .
$$

(This is also true when $\mathcal{P}_{N}(0) e_{j}$ is replaced by $\mathcal{W}_{N}(0) e_{j}$ in the above statement.) 
Indeed, one can see from (1.10) and (5.3) that for each $j \geq 1$ and $t \geq 0$,

$$
\left\{\begin{aligned}
& \mathcal{P}_{N}(t)\left(\mathcal{P}_{N}(0) e_{j}\right)= e^{-t \eta_{j}}\left(1+\sum_{l=0}^{N-1} p_{l}(t) \eta_{j}^{-l-1}\right)\left(1+\sum_{l=0}^{N-1} p_{l}(0) \eta_{j}^{-l-1}\right) e_{j}, \\
& \mathcal{W}_{N}(t)\left(\mathcal{P}_{N}(0) e_{j}\right)=\left(\sum_{l=0}^{N-1} h_{l}(t) \eta_{j}^{-l-1}\right)\left(1+\sum_{l=0}^{N-1} p_{l}(0) \eta_{j}^{-l-1}\right) e_{j}, \\
& \mathfrak{R}_{N}(t)\left(\mathcal{P}_{N}(0) e_{j}\right)=\left(h_{N}(t) \eta_{j}^{-N-1}+p_{N}(t) e^{-t \eta_{j}} \eta_{j}^{-N-1}+R_{N+1}\left(t, \eta_{j}\right) \eta_{j}^{-N-2}\right) \\
&\left(1+\sum_{l=0}^{N-1} p_{l}(0) \eta_{j}^{-l-1}\right) e_{j} .
\end{aligned}\right.
$$

Since $\lim _{j \rightarrow+\infty} \eta_{j}=+\infty$, the above, along with the first inequality in (5.2) and the estimate (3.3), implies

$$
\mathcal{P}_{N}(\cdot) e_{j}, \mathcal{W}_{N}(\cdot) e_{j}, \Re_{N}(\cdot) e_{j} \not \equiv 0 \text { for large } j \in \mathbb{N}^{+}
$$

At the same time, the three functions on the left hand side above are analytic from $\mathbb{R}^{+}$ to $L^{2}(\Omega)$. (For the first two functions, their analyticity follows from (1.10) and (5.1) and then the analyticity of the last one is derived from Proposition 4.9.) Therefore, they are non-trivial almost everywhere. This is exactly (5.4). (In a similar way, (5.4) can be verified in the case that $\mathcal{P}_{N}(0) e_{j}$ is replaced by $\mathcal{W}_{N}(0) e_{j}$.)

\section{Conclusions and further comments}

In this paper we have presented a decomposition for the flow generated by the equation (1.1), which reveals the hybrid parabolic-hyperbolic behavior of the flow. We have also described the nature of each of the components in the decomposition; that has been illustrated through an example.

A number of interesting issues could be considered in connection with the results and methods developed in this paper. Here, we briefly give some of them.

- Smooth memory kernels. It would be interesting to analyze whether (1.11), (1.12) and (1.14) hold under the assumption that $M \in C^{\infty}\left(\overline{\mathbb{R}^{+}}\right) \backslash\{0\}$.

- Decomposition with infinite series. It would be interesting to obtain a meaningful decomposition without the intervention of the third remainder term.

- Space-dependent memory kernels. The extension of the results of this paper to the spacedependent memory kernels $M=M(t, x)$ is open.

- Memory kernels in the principal part of the model. It would be interesting to extend our decomposition and analysis to the following two types of heat equations with memory kernels:

(i) $\partial_{t} y-\Delta y-\int_{0}^{t} M(t-s) \Delta y(s) d s=0$

(ii) $\partial_{t} y-\int_{0}^{t} M(t-s) \Delta y(s) d s=0$, 
that are more relevant from an applied and modelling viewpoint.

- Other equations with memory. It would be interesting to extend this decomposition to other models such as wave equations with memory kernels.

\section{Appendix}

Proposition 7.1. For each $t \geq 0$ and $s \in \mathbb{R}$, it holds that

$$
\Phi(t) y_{0}=y\left(t ; y_{0}\right), y_{0} \in \mathcal{H}^{s}
$$

and that $\Phi(t)$ belongs to $\mathcal{L}\left(\mathcal{H}^{s}\right)$.

Proof. Arbitrarily fix $s \in \mathbb{R}$. Since $\left\{e^{t A}\right\}_{t \geq 0}$ is a $C_{0}$ semigroup over $\mathcal{H}^{s}$, we can use a standard method (see for instance [18, Theorem 1.2 in Section 6.1, p. 184]) to show that for each $y_{0} \in$ $\mathcal{H}^{s}$, the solution $y\left(\cdot ; y_{0}\right)$ belongs to the space $C\left(\overline{\mathbb{R}^{+}} ; \mathcal{H}^{s}\right)$. This, along with (1.2), yields (7.1). Moreover, one can directly check that for each $t \geq 0, \Phi(t) \in \mathcal{L}\left(\mathcal{H}^{s}\right)$. This completes the proof.

The proof of Lemma 2.1 is given as follows.

Proof of Lemma 2.1. By standard density arguments, it suffices to show that for each $k \in \mathbb{N}$, the following property $\left(\mathcal{E}_{k}\right)$ is true: For all kernels $\left\{M_{l}\right\}_{l=1}^{j} \subset C^{\infty}\left(\overline{\mathbb{R}^{+}}\right)$,

$$
\left|\frac{d^{k}}{d t^{k}} M_{1} * \cdots * M_{j}(t)\right| \leq \mathcal{C}(j, k, t) \prod_{l \leq q(j, k)}\left\|M_{l}\right\|_{C^{p(j, k)}([0, t])} \prod_{l>q(j, k)}\left\|M_{l}\right\|_{C^{p(j, k)-1}([0, t])}, \quad t>0 .
$$

Here and in what follows, we set $\prod_{\emptyset} \cdot:=1$ and let

$$
\left\{\begin{aligned}
p(j, k) & :=\max \{k-j+1,1\} \\
q(j, k) & :=\min \{k, j\} \\
\mathcal{C}(j, k, t) & :=\sum_{l=j-1-k}^{j-1} \chi_{\mathbb{N}}(l) \frac{t^{l}}{l !}, \quad t>0 .
\end{aligned}\right.
$$

Now we will use the induction to prove the above $\left(\mathcal{E}_{k}\right)$ for each $k \in \mathbb{N}$. To this end, we first check $\left(\mathcal{E}_{0}\right)$. Indeed, for each $j \in \mathbb{N}^{+}$and each $\left\{M_{l}\right\}_{l=1}^{j} \subset C^{\infty}\left(\overline{\mathbb{R}^{+}}\right)$, we have that

$$
\left|M_{1} * \cdots * M_{j}(t)\right| \leq\left(\prod_{l=1}^{j}\left\|M_{l}\right\|_{C([0, t])}\right) \int_{0}^{t} \int_{0}^{t_{1}} \cdots \int_{0}^{t_{j-1}} d t_{j} \cdots d t_{1}=\frac{t^{j-1}}{(j-1) !} \prod_{l=1}^{j}\left\|M_{l}\right\|_{C([0, t])} .
$$

This, along with (7.3), leads to (7.2) with $k=0$. Therefore $\left(\mathcal{E}_{0}\right)$ is true.

Next, we will show $\left(\mathcal{E}_{k_{0}+1}\right)$ for any $k_{0} \in \mathbb{N}$, under the assumption that $\left(\mathcal{E}_{k}\right)$ holds for all $k \leq k_{0}$. For this purpose, we arbitrarily fix $k_{0} \in \mathbb{N}, j \in \mathbb{N}^{+}$and $\left\{M_{l}\right\}_{l=1}^{j} \subset C^{\infty}\left(\overline{\mathbb{R}^{+}}\right)$. Since 
(7.2), with $j=1$, holds clearly, we only need to focus on the situation that $j \geq 2$. There are only two possibilities for $j$ : either $k_{0} \leq j-1$ or $k_{0} \geq j$.

In the case when $k_{0} \leq j-1$, we have three observations: First, by direct computations, we find

$$
\begin{aligned}
\frac{d^{k_{0}+1}}{d t^{k_{0}+1}} M_{1} * \cdots * M_{j} & =\frac{d^{k_{0}}}{d t^{k_{0}}}\left(\frac{d}{d t} M_{1} * \cdots * M_{j}\right) \\
& =\frac{d^{k_{0}}}{d t^{k_{0}}}\left(M_{1}(0) M_{2} * \cdots * M_{j}+M_{1}^{\prime} * M_{2} * \cdots * M_{j}\right) \\
& =M_{1}(0) \frac{d^{k_{0}}}{d t^{k_{0}}} M_{2} * \cdots * M_{j}+\frac{d^{k_{0}}}{d t^{k_{0}}} M_{2} * \cdots * M_{j} * M_{1}^{\prime}
\end{aligned}
$$

Second, we apply $\left(\mathcal{E}_{k_{0}}\right)$ twice to find that for each $t>0$,

$$
\begin{gathered}
\left|\frac{d^{k_{0}}}{d t^{k_{0}}} M_{2} * \cdots * M_{j}(t)\right| \leq \mathcal{C}\left(j-1, k_{0}, t\right)\left(\prod_{2 \leq l \leq k_{0}+1}\left\|M_{l}\right\|_{C^{1}([0, t])}\right)\left(\prod_{l>k_{0}+1}\left\|M_{l}\right\|_{C([0, t])}\right) ; \quad(7.5) \\
\left|\frac{d^{k_{0}}}{d t^{k_{0}}} M_{2} * \cdots * M_{j} * M_{1}^{\prime}(t)\right| \leq \mathcal{C}\left(j, k_{0}, t\right)\left(\prod_{2 \leq l \leq k_{0}+1}\left\|M_{l}\right\|_{C^{1}([0, t])}\right)\left(\prod_{l>k_{0}+1}\left\|M_{l}\right\|_{C([0, t])}\right)\left\|M_{1}^{\prime}\right\|_{C([0, t])} .
\end{gathered}
$$

Third, from the third definition in (7.3), we see

$$
\max \left\{\mathcal{C}\left(j-1, k_{0}, t\right), \mathcal{C}\left(j, k_{0}, t\right)\right\} \leq \mathcal{C}\left(j, k_{0}+1, t\right), \quad t>0 .
$$

Now, from (7.4), (7.5), (7.6) and (7.7), it follows that for each $t>0$,

$$
\begin{aligned}
\left|\frac{d^{k_{0}+1}}{d t^{k_{0}+1}} M_{1} * \cdots * M_{j}(t)\right| \leq \mathcal{C}(j, & \left.k_{0}+1, t\right)\left(\left\|M_{1}\right\|_{C([0, t])}+\left\|M_{1}^{\prime}\right\|_{C([0, t])}\right) \\
& \times\left(\prod_{2 \leq l \leq k_{0}+1}\left\|M_{l}\right\|_{C^{1}([0, t])}\right)\left(\prod_{l>k_{0}+1}\left\|M_{l}\right\|_{C([0, t])}\right) .
\end{aligned}
$$

This, along with (7.3), leads to $(7.2)$ (with $k=k_{0}+1 \leq j$ ). Therefore, $\left(\mathcal{E}_{k_{0}+1}\right)$ is true when $k_{0} \leq j-1$.

In the case that $k_{0} \geq j$, we see from (7.3) that

$$
\left\{\begin{array}{l}
p\left(j, k_{0}+1\right)=p\left(j-1, k_{0}\right)=p\left(j, k_{0}\right)+1=k_{0}-j+2 \\
q\left(j, k_{0}+1\right)=q\left(j, k_{0}\right)=q\left(j-1, k_{0}\right)+1=j .
\end{array}\right.
$$

Then by the similar arguments as those in (7.4)-(7.7), one can get that for each $t>0$,

$$
\begin{aligned}
&\left|\frac{d^{k_{0}+1}}{d t^{k_{0}+1}} M_{1} * \cdots * M_{j}(t)\right| \leq \mathcal{C}\left(j, k_{0}+1, t\right)\left(\left\|M_{1}\right\|_{C([0, t])}+\left\|M_{1}^{\prime}\right\|_{C^{p\left(j, k_{0}\right)}([0, t])}\right) \\
& \quad \times \prod_{l=2}^{j}\left\|M_{l}\right\|_{C^{p\left(j-1, k_{0}\right)}([0, t])} \\
&=\mathcal{C}\left(j, k_{0}+1, t\right) \prod_{l=1}^{j}\left\|M_{l}\right\|_{C^{p\left(j, k_{0}+1\right)}([0, t])} .
\end{aligned}
$$


This, together with (7.3), leads to $(7.2)$ (with $k=k_{0}+1>j$ ). Therefore, $\left(\mathcal{E}_{k_{0}+1}\right)$ holds in the case that $k_{0} \geq j$.

Hence, we complete the proof of Lemma 2.1.

Acknowledgments. The authors would like to gratefully thank Dr. Huaiqiang Yu and Dr. Christophe Zhang for their valuable comments and suggestions.

The first and second authors were supported by the National Natural Science Foundation of China under grants 11971022,11801408 and 12171359 .

The third author has been funded by the Alexander von Humboldt-Professorship program, the European Research Council (ERC) under the European Union's Horizon 2020 research and innovation programme (grant agreement No. 694126-DyCon), the Transregio 154 Project "Mathematical Modeling, Simulation and Optimization Using the Example of Gas Networks" of the German DFG, the grant MTM2017-92996 of MINECO (Spain), ELKARTEK project KK-2018/00083 ROAD2DC of the Basque Government and the Marie Sklodowska-Curie grant agreement No. 765579-ConFlex.

\section{References}

[1] G. Amendola, M. Fabrizio and J. Golden. Thermodynamics of materials with memory: Theory and applications. Springer, New York, 2012.

[2] L. Boltzmann. Zur theorie der elastischen nachwirkung. Wien. Ber. 70 (1874) 275-306.

[3] L. Boltzmann. Zur theorie der elastischen nachwirkung. Wien. Ber. 5 (1878) 430-432.

[4] C. Cattaneo. A form of heat conduction equation which eliminates the paradox of instantaneous propagation. Compute. Rendus. 247 (1958) 431-433.

[5] F. Chaves-Silva, X. Zhang and E. Zuazua. Controllability of evolution equations with memory. SIAM J. Control Optim. 55 (2017) 2437-2459.

[6] R. Christensen. Theory of viscoelasticity, an introduction. Academic Press, New York, 1982.

[7] B. Coleman and M. Gurtin. Equipresence and constitutive equations for rigid heat conductors. Z. Angew. Math. Phys. 18 (1967) 199-208.

[8] C. Dafermos. Asymptotic Stability in Viscoelasticity. Arch. Ration. Mech. Anal. 37 (1970) 297-308.

[9] M. Fabrizio, C. Giorgi and V. Pata. A new approach to equations with memory. Arch. Ration. Mech. Anal. 198 (2010) 189-232.

[10] X. Fu, J. Yong and X. Zhang. Controllability and observability of the heat equations with hyperbolic memory kernel. J. Differential Equations 247 (2009) 2395-2439.

[11] M. Gurtin and A. Pipkin. A general theory of heat conduction with finite wave speeds. Arch. Ration. Mech. Anal. 31 (1968) 113-126.

[12] L. Hörmander. The Analysis of Linear Partial Differential Operators, Vol. 3, SpringerVerlag, 2007.

[13] Q. Lü, X. Zhang and E. Zuazua. Null controllability for wave equations with memory. J. Math. Pures Appl. 108 (2017) 500-531. 
[14] J. Maxwell. On the dynamical theory of gases. Phil. Trans. Roy. Soc. London 157 (1867) $49-88$.

[15] M. Reed and B. Simon. Methods of Modern Mathematical Physics I: Functional Analysis, Academic Press, 1980.

[16] M. Reed and B. Simon. Methods of Modern Mathematical Physics IV: Analysis of Operators, Academic Press, 1978.

[17] L. Pandolfi. Linear systems with persistent memory: An overview of the biblography on controllability. arXiv: 1804.01865v1 (2018), preprint.

[18] A. Pazy. Semigroups of Linear Operators and Application to Partial Differential Equations. Springer-Verlag, New York, Inc., 1983.

[19] V. Volterra. Sur les équations intégro-différentielles et leurs applications. Acta Math. 35 (1912) 295-356.

[20] V. Volterra. Leçons sur les fonctions de lignes. Gauthier-Villars, Paris, 1913.

[21] G. Wang, Y. Zhang and E. Zuazua. Reachable subspaces, control regions and heat equations with memory. arXiv:2101.10615v1, preprint. 